\title{
ANALYSIS OF A SEQUENTIAL REGULARIZATION METHOD FOR THE UNSTEADY NAVIER-STOKES EQUATIONS
}

\author{
XILIANG LU, PING LIN, AND JIAN-GUO LIU
}

\begin{abstract}
The incompressibility constraint makes Navier-Stokes equations difficult. A reformulation to a better posed problem is needed before solving it numerically. The sequential regularization method (SRM) is a reformulation which combines the penalty method with a stabilization method in the context of constrained dynamical systems and has the benefit of both methods. In the paper, we study the existence and uniqueness for the solution of the SRM and provide a simple proof of the convergence of the solution of the SRM to the solution of the Navier-Stokes equations. We also give error estimates for the time discretized SRM formulation.
\end{abstract}

\section{INTRODUCTION}

We consider the unsteady Navier-Stokes equations with homogeneous Dirichlet boundary condition:

$$
\begin{aligned}
& \mathbf{u}_{t}+(\mathbf{u} \cdot \operatorname{grad}) \mathbf{u}=\nu \Delta \mathbf{u}-\operatorname{grad} p+\mathbf{f}, \\
& \operatorname{div} \mathbf{u}=0, \\
& \left.\mathbf{u}\right|_{\partial \Omega}=\mathbf{0},\left.\mathbf{u}\right|_{t=0}=\mathbf{u}_{0},
\end{aligned}
$$

in a bounded domain $\Omega$ and a time interval $0 \leq t \leq T$. Here $\mathbf{u}(\mathbf{x}, t)$ represents the velocity of a viscous incompressible fluid, $p(\mathbf{x}, t)$ represents the pressure, $\mathbf{f}$ the prescribed external force, $\mathbf{u}_{0}(\mathbf{x})$ the prescribed initial velocity, and with no-slip velocity boundary condition.

A lot of methods have been proposed for the numerical solution of the NavierStokes equation. The equation is an infinite-dimensional constrained dynamical system (see [19, 10, 7]), where the incompressibility condition (1.2) can be seen as a constraint and the pressure $p$ as the Lagrange multiplier. The special role of the pressure $p$ leads to computational difficulties, and a direct discretization to the system is not recommended. A reformulation or regularization is thus needed to obtain a better behaved problem, where a direct discretization may then be applied. An important structural property of a reformulation is the stability of the associated constraint or invariant set in the dynamical system context. A simple stabilization formulation is the Baumgarte stabilization (see [4]), which is also called an ambientspace (impetus-striction) formulation in 7]. The method basically replaces (1.2)

Received by the editor July 25, 2006 and, in revised form, June 4, 2007.

2000 Mathematics Subject Classification. Primary 65M12; Secondary 76D05.

Key words and phrases. Navier-Stokes equations, iterative penalty method, implicit parabolic PDE, error estimates, constrained dynamical system, stabilization method.

The research was supported by several grants at the Department of Mathematics, National University of Singapore.

(C)2008 American Mathematical Society
Reverts to public domain 28 years from publication 1467 
by

$$
\alpha_{1}(\operatorname{div} \mathbf{u})_{t}+\alpha_{2} \operatorname{div} \mathbf{u}=0
$$

Under this formulation the invariant set $\operatorname{div} \mathbf{u}=0$ is exponentially attractive if $\alpha_{1}, \alpha_{2}>0$. However, when coupled with the momentum equation (1.1) a Poisson equation for the pressure has to be solved and it is well known that an artificial boundary condition for the pressure has to be imposed. Also, variables $\mathbf{u}$ and $p$ are coupled. On the other hand, another reformulation, the penalty method (cf. [15, 17, 10]), is simple and, in particular, it does not require the boundary condition for the pressure, and the variables $\mathbf{u}$ and $p$ are uncoupled. However, it introduces a penalty parameter $\epsilon$, which has to be very small to maintain the accuracy of the penalized system. This small parameter $\epsilon$ gives a sharp initial layer in the pressure and causes some inaccuracy in the pressure near the beginning of time if the initial pressure is not zero. Also, the error bound of a spatial discretization will be inversely proportional to the small parameter $\epsilon$ (cf. [14]) in general, which could be useless as $\epsilon$ is required to be very small in the formulation.

In [10, a sequential regularization method (SRM) is proposed and analyzed, based on methods of dealing with differential algebraic equations (see, e.g. [2, 3]). The method is a combination of the penalty method and Baumgarte stabilization and can be seen as an iterative penalty method or a modified augmented Lagrangian method for nonstationary problems (cf. [6]). The method is defined as follows: with $p_{0}(\mathbf{x}, t)$ an initial guess, given a small parameter $\epsilon$ and two nonnegative constants $\alpha_{1}, \alpha_{2}$, for $s=1,2, \ldots$, solve the problem

$$
\begin{aligned}
& \left(\mathbf{u}_{s}\right)_{t}+\left(\mathbf{u}_{s} \cdot \operatorname{grad}\right) \mathbf{u}_{s}=\nu \Delta \mathbf{u}_{s}-\operatorname{grad} p_{s}+\mathbf{f}, \\
& \operatorname{div}\left(\alpha_{1}\left(\mathbf{u}_{s}\right)_{t}+\alpha_{2} \mathbf{u}_{s}\right)=\epsilon\left(p_{s-1}-p_{s}\right), \\
& \left.\mathbf{u}_{s}\right|_{\partial \Omega}=\mathbf{0},\left.\mathbf{u}_{s}\right|_{t=0}=\mathbf{u}_{0} .
\end{aligned}
$$

If we choose $\alpha_{1}=0, \alpha_{2}=1, s=1$ and $p_{0}=0$, the SRM is exactly the penalty method. We are interested in the SRM because it keeps the benefits of the penalty method, but unlike the penalty method, the parameter $\epsilon$ is not necessarily very small, and thus the reformulated system is more stable or less stiff (see [10] and the convergence estimate later in Section 2). Furthermore, it approximates the divergence condition better than the penalty method. We can simply see from (1.5) that the divergence of the velocity is of $O\left(\epsilon^{s}\right)$ and this bound is independent of the time $t$ if $p_{s-1}-p_{s}=O\left(\epsilon^{s-1}\right)$, which we can show later. The SRM may also avoid the initial layer in the pressure variable (see some explanation in 2, 3] ). Since it does not require $\epsilon$ to be very small, it would be fine even if the error bound of the finite element method is inversely proportional to $\epsilon$. So any standard finite elements can possibly be used in numerical computation. In addition, under this formulation, a fully explicit method can possibly be designed (see [12]).

In this paper we will take $\alpha_{1}=1$ and $\alpha_{2}=\alpha>0$. The domain $\Omega$ will be assumed to be smooth and bounded in $R^{2}$ (a remark will be given for the 3D case). At each SRM iteration, after eliminating $p_{s}$ from (1.4), (1.5), omitting the index $s$, and redefining the right-hand side as $\mathbf{f}$, we need to solve a PDE of the form

$$
\begin{aligned}
& \mathbf{u}_{t}-\frac{1}{\epsilon} \operatorname{grad} \operatorname{div}\left(\mathbf{u}_{t}+\alpha \mathbf{u}\right)-\nu \Delta \mathbf{u}+(\mathbf{u} \cdot \operatorname{grad}) \mathbf{u}=\mathbf{f}, \\
& \left.\mathbf{u}\right|_{\partial \Omega}=0,\left.\mathbf{u}\right|_{t=0}=\mathbf{u}_{0} .
\end{aligned}
$$


This PDE is an implicit parabolic equation, the operator $I-\frac{1}{\epsilon}$ grad div is a degenerate elliptic operator, and we will show the existence of the solution to this PDE in this paper.

From the formulation point of view we may find that the SRM is a more natural formulation than the penalty formulation for the unsteady Navier-Stokes equations. To see this let us consider the time-independent Stokes equations with a perturbation in the incompressibility condition (i.e., a steady-state inhomogeneous Stokes equation)

$$
\begin{aligned}
& -\nu \triangle \mathbf{u}+\operatorname{grad} p=\mathbf{f}, \\
& \operatorname{divu}=g, \\
& \left.\mathbf{u}\right|_{\partial \Omega}=0 .
\end{aligned}
$$

If the basic compatibility holds $\left(\int_{\Omega} g=0\right)$, we have

$$
\|\triangle \mathbf{u}\|+\|\operatorname{grad} p\| \leq C_{0}(\|\mathbf{f}\|+\|\operatorname{grad} g\|),
$$

where $C_{0}$ only depends on the domain $\Omega$ and $\nu$ (see [18]). When we try to estimate the error for the penalty method (or iterative penalty method) for the timeindependent problem, this estimation plays a crucial role. When the time is taken into account, i.e. considering

$$
\begin{aligned}
& \mathbf{u}_{t}-\nu \triangle \mathbf{u}+\operatorname{grad} p=\mathbf{f} \\
& \operatorname{div} \mathbf{u}=g, \\
& \left.\mathbf{u}\right|_{\partial \Omega}=0,\left.\mathbf{u}\right|_{t=0}=\mathbf{u}_{0},
\end{aligned}
$$

where the basic compatibility holds $\left(\int_{\Omega} g=0\right.$, div $\left.\mathbf{u}_{0}=g(0)\right)$, we can obtain (see [13])

$$
\begin{aligned}
& \sup _{0 \leq t \leq T}\|\mathbf{u}\|_{1}^{2}+\int_{0}^{T}\left(\|\triangle \mathbf{u}\|^{2}+\|\operatorname{grad} p\|^{2}\right) d t \\
& \quad \leq C\left(\left\|\mathbf{u}_{0}\right\|_{1}^{2}+\int_{0}^{T}\|\mathbf{f}\|^{2}+\|\operatorname{grad} g\|^{2}+\left\|g_{t}\right\|_{\left(H^{1}\right)^{\prime}}^{2} d t\right),
\end{aligned}
$$

where $\left(H^{1}\right)^{\prime}$ is the dual space of $H^{1}$. If we apply the penalty method directly to the time-dependent Navier-Stokes equation, then the last term on the right-hand side of the above inequality will depend on the time derivative of the pressure (the extra requirement for the pressure reads $p \in H^{1}\left(0, T,\left(H^{1}\right)^{\prime}\right)$. It is not trivial to obtain the estimation of $p_{t}$ for the Navier-Stokes equations. In general the global compatibility condition is required (see [9]). After iterations, things will be even worse, where the regularity for higher order derivatives of $p$ with respect to $t$ may be needed. From this point of view, we may not expect a simple iterative penalty scheme to significantly improve its accuracy. However, if we modify the inhomogeneous time-dependent Stokes equation to what the SRM is based on, then

$$
\begin{array}{r}
\mathbf{u}_{t}-\nu \triangle \mathbf{u}+\operatorname{grad} p=\mathbf{f}, \\
\operatorname{div} \mathbf{u}_{t}+\alpha \operatorname{divu}=g, \\
\left.\mathbf{u}\right|_{\partial \Omega}=0,\left.\quad \mathbf{u}\right|_{t=0}=\mathbf{u}_{0} .
\end{array}
$$

If $\int_{\Omega} g=0$ and $\operatorname{div} \mathbf{u}_{0}=0$, we have (see [10])

$$
\begin{aligned}
\sup _{0 \leq t \leq T} & \|\mathbf{u}\|_{1}^{2}+\int_{0}^{T}\left(\|\triangle \mathbf{u}\|^{2}+\|\operatorname{grad} p\|^{2}\right) d t \\
\leq & C\left(\left\|\mathbf{u}_{0}\right\|_{1}^{2}+\int_{0}^{T}\|\mathbf{f}\|^{2}+\|\operatorname{grad} g\|^{2} d t\right),
\end{aligned}
$$


where the constant $C$ only depends on $\nu, \alpha$, the domain $\Omega$ and the time length $T$. The Navier-Stokes system is an index-2 partial differential algebraic system (see [10]) and ill-posed in time in the sense that the solution depends on $g_{t}$ if the equation (1.2) is perturbed by a function $g$. From estimation (1.11), we can see that the solution of the sequential regularization reformulation is well posed (or more stable in time) since it does not depend on $g_{t}$. Using this estimation, we can obtain an error bound for the SRM similar to that for the time-independent Navier-Stokes equations.

The paper is organized as follows. In $\S 2$, we describe the sequential regularization formulation. A modified convergence theorem is proved using a simpler method than in [10. In $\S 3$, we prove the existence of the solution of equation (1.7), (1.8). In $\S 4$, we discretize the regularized problems in the temporal direction and analyze the convergence of a number of semi-discrete schemes. Numerical experiments which show the SRM works well may be found in [10, 11, 12, 14.

\section{Preliminaries, the Sequential Regularization method AND ITS CONVERGENCE}

We first describe some notation and assumptions. As usual, we use $\mathbf{L}^{p}(\Omega)$, or simply $\mathbf{L}^{p}$, to denote the space of $p$ th-power integrable functions in $\Omega$, and

$$
\|\mathbf{u}\|_{\mathbf{L}^{p}}=\left(\int_{\Omega} \sum_{i=1}^{n}\left|u_{i}\right|^{p} d \mathbf{x}\right)^{\frac{1}{p}}
$$

its norm, where $\mathbf{u}=\left(u_{1}, \cdots, u_{n}\right)$. We denote the inner product in $\mathbf{L}^{2}$ by $(\cdot, \cdot)$ and let $\|\cdot\| \equiv\|\cdot\|_{\mathbf{L}^{2}} \cdot \mathbf{H}^{m}$ includes functions which are square integrable up to $m$ th derivatives with norm

$$
\|\mathbf{u}\|_{\mathbf{H}^{m}}=\left(\sum_{0 \leq|\alpha| \leq m}\left\|D^{\alpha} \mathbf{u}\right\|^{2}\right)^{\frac{1}{2}}
$$

For simplicity, let $\|\cdot\|_{m} \equiv\|\cdot\|_{\mathbf{H}^{m}}$. For a normed linear space $B$ with norm $\|\cdot\|_{B}$ and a sufficiently regular function $g:[\alpha, \beta] \rightarrow B$, we define

$$
\|g\|_{L^{2}([\alpha, \beta] ; B)}=\left(\int_{\alpha}^{\beta}\|g(\cdot, t)\|_{B}^{2} d t\right)^{\frac{1}{2}} \text { and }\|g\|_{L^{\infty}([\alpha, \beta] ; B)}=\sup _{\alpha \leq t \leq \beta}\|g(\cdot, t)\|_{B} .
$$

If $[\alpha, \beta]=[0, T]$, we simplify the notation as $\|g\|_{L^{2}(B)}$ and $\|g\|_{L^{\infty}(B)}$, respectively.

We list some widely used inequalities here, which may be found in many analysis textbooks (see $[5$ ). We define $C$ as a generic constant which does not depend on the choice of functions.

- Poincaré's inequality:

$$
\|u\| \leq C\|\operatorname{grad} u\|, u \in H_{0}^{1}
$$

or, more generally, for $u \in H^{1}$,

$$
\|u\| \leq C\left(\|\operatorname{grad} u\|+\left|\int_{\Omega} u d x\right|\right) .
$$

- Hölder's inequality:

$$
\int_{\Omega}|f g h| \leq C\|f\|_{L^{p}}\|g\|_{L^{q}}\|h\|_{L^{r}}
$$

where $p, q, r>1$ and $\frac{1}{p}+\frac{1}{q}+\frac{1}{r}=1$. 
- Sobolev's inequality:

$$
\|u\|_{L^{q}} \leq C\|u\|_{m},
$$

if $\frac{1}{2}-\frac{m}{n}>0$ and $\frac{1}{q}=\frac{1}{2}-\frac{m}{n}$, where $n$ is the dimension of the domain $\Omega$

$$
\|u\|_{L^{\infty}} \leq C\|u\|_{m}
$$

if $\frac{1}{2}-\frac{m}{n}<0$, where $n$ is the dimension of the domain $\Omega$;

$$
\|u\|_{L^{4}} \leq C\|u\|^{\frac{1}{2}}\|u\|_{1}^{\frac{1}{2}}
$$

where the domain is in $R^{2}$.

- Young's inequality:

$$
a b \leq \epsilon a^{p}+c_{\epsilon} b^{q},
$$

where $1<p<\infty, \epsilon>0, \frac{1}{p}+\frac{1}{q}=1, c_{\epsilon}$ is a constant which only depends on $\epsilon$.

- Gronwall's inequality in differential form.

Let $y(t)$ be a nonnegative, absolutely continuous function in $[0, t]$ that satisfies, for almost every $t$, the differential inequality:

$$
y^{\prime}(t) \leq a(t) y(t)+b(t),
$$

where $a(t)$ and $b(t)$ are nonnegative, summable functions in $[0, t]$. Then we have:

$$
y(t) \leq e^{\int_{0}^{t} a(s) d s}\left[y(0)+\int_{0}^{t} b(s) d s\right] .
$$

- Discrete Gronwall's inequality.

Let $y^{n}, a^{n}, b^{n}$ and $c^{n}$ be nonnegative sequences, satisfying

$$
y^{n}+\triangle t \sum_{i=0}^{n} a^{i} \leq y^{0}+\triangle t \sum_{i=0}^{n}\left(b^{i} y^{i}+c^{i}\right),
$$

with $\triangle t b^{i}<1$. Then we have

$$
y^{n}+\triangle t \sum_{i=1}^{n} a^{i} \leq e^{C \triangle t \sum_{i=1}^{n} b^{i}}\left(\triangle t \sum_{i=1}^{n} c^{i}+y^{0}\right),
$$

where $C=\max _{0 \leq i \leq n}\left(1-\triangle t b^{i}\right)^{-1}$.

We are interested in the case that the problem (1.1), (1.2), (1.3) has a unique solution and the solution satisfies

$$
\|\mathbf{u}\|_{1}^{2}+\int_{0}^{T}\left(\left\|\mathbf{u}_{t}\right\|^{2}+\|\mathbf{u}\|_{2}^{2}+\|p\|_{1}^{2}\right) d t \leq M,
$$

where the pressure $p$ is up to an arbitrary constant which is determined by

$$
\int_{\Omega} p(\mathbf{x}, \cdot) d \mathbf{x}=0 .
$$

Hence, some basic compatibility condition has to be assumed (cf. [9]):

$$
\left.\mathbf{u}_{0}\right|_{\partial \Omega}=\mathbf{0}, \operatorname{div} \mathbf{u}_{0} \equiv 0 .
$$


Furthermore, we assume

$$
\sup _{t \in[0, T]}\|\mathbf{f}\| \leq M_{1},\left\|\mathbf{u}_{0}\right\|_{2} \leq M_{1}
$$

where $M_{1}$ is a positive constant.

Eliminating $p_{s}$ from equations (1.4), (1.5) (with $\alpha_{1}=1, \alpha_{2}=\alpha$ ), we can reformulate the SRM equation (cf. [10]) as follows: with $p_{0}(\mathbf{x}, t)$ an initial guess, for $s=1,2, \cdots$, solve the problem

$$
\begin{aligned}
& \left(\mathbf{u}_{s}\right)_{t}-\frac{1}{\epsilon} \operatorname{grad}\left(\left(\operatorname{div} \mathbf{u}_{s}\right)_{t}+\alpha \operatorname{div} \mathbf{u}_{s}\right)+\left(\mathbf{u}_{s} \cdot \operatorname{grad}\right) \mathbf{u}_{s} \\
& =\nu \Delta \mathbf{u}_{s}-\operatorname{grad} p_{s-1}+\mathbf{f}, \\
& \left.\mathbf{u}_{s}\right|_{\partial \Omega}=\mathbf{0},\left.\mathbf{u}_{s}\right|_{t=0}=\mathbf{u}_{0}, \\
& p_{s}=p_{s-1}-\frac{1}{\epsilon}\left(\left(\operatorname{div} \mathbf{u}_{s}\right)_{t}+\alpha \operatorname{div} \mathbf{u}_{s}\right) .
\end{aligned}
$$

We take $p_{0}$ satisfying (2.13). Then it is easy to see that $p_{s}$ satisfies (2.13) for all $s$.

To simplify the nonlinear term, we introduce a few operators and inequalities (see [18, 19]):

$$
\begin{array}{cl}
B(\mathbf{u}, \mathbf{v})=(\mathbf{u} \cdot \operatorname{grad}) \mathbf{v}, & \bar{B}(\mathbf{u}, \mathbf{v})=B(\mathbf{u}, \mathbf{v})+\frac{1}{2}(\operatorname{div} \mathbf{u}) \mathbf{v}, \\
b(\mathbf{u}, \mathbf{v}, \mathbf{w})=(B(\mathbf{u}, \mathbf{v}), \mathbf{w}), & \bar{b}(\mathbf{u}, \mathbf{v}, \mathbf{w})=(\bar{B}(\mathbf{u}, \mathbf{v}), \mathbf{w}) .
\end{array}
$$

We can easily check that

$$
\bar{b}(\mathbf{u}, \mathbf{v}, \mathbf{w})=\frac{1}{2}\{b(\mathbf{u}, \mathbf{v}, \mathbf{w})-b(\mathbf{u}, \mathbf{w}, \mathbf{v})\}, \quad \forall \mathbf{u}, \mathbf{v}, \mathbf{w} \in \mathbf{H}_{0}^{1}(\Omega) .
$$

Therefore,

$$
\bar{b}(\mathbf{u}, \mathbf{v}, \mathbf{v})=0, \quad \forall \mathbf{u}, \mathbf{v} \in \mathbf{H}_{0}^{1}(\Omega) .
$$

For trilinear forms $b$ (or $\bar{b}$ ), we can prove the following inequality by a combination of integration of parts, Hölder's and Sobolev's inequalities (see for instance [19]).

Lemma 2.1. Assume $\Omega \in R^{n}$. Then the trilinear form $b$ (or $\bar{b}$ ) is defined as a bounded linear functional on $\mathbf{H}^{m_{1}} \times \mathbf{H}^{m_{2}+1} \times \mathbf{H}^{m_{3}}$, where $m_{i} \geq 0$ and

$$
\begin{aligned}
m_{1}+m_{2}+m_{3} & \geq \frac{n}{2} \text { if } m_{i} \neq \frac{n}{2}, \\
m_{1}+m_{2}+m_{3}>\frac{n}{2} \text { if } m_{i} & =\frac{n}{2} \text { for some } i .
\end{aligned}
$$

Lemma 2.2. As a corollary of Lemma 2.1, we choose proper $m_{i}$ to get:

$$
b(\mathbf{u}, \mathbf{v}, \mathbf{w}) \leq C\left\{\begin{array}{l}
\|\mathbf{u}\|_{1}\|\mathbf{v}\|_{1}\|\mathbf{w}\|_{1} \\
\|\mathbf{u}\|_{2}\|\mathbf{v}\|_{1}\|\mathbf{w}\| \\
\|\mathbf{u}\|_{1}\|\mathbf{v}\|_{2}\|\mathbf{w}\| .
\end{array}\right.
$$

Moreover, if $\Omega \in R^{2}$, we have:

$$
\begin{aligned}
& |b(\mathbf{u}, \mathbf{v}, \mathbf{w})| \leq C\|\mathbf{u}\|^{\frac{1}{2}}\|\mathbf{u}\|_{1}^{\frac{1}{2}}\|\mathbf{v}\|_{1}^{\frac{1}{2}}\|\mathbf{v}\|_{2}^{\frac{1}{2}}\|\mathbf{w}\|, \quad \forall \mathbf{v} \in \mathbf{H}^{2} \cap \mathbf{H}_{0}^{1}, \mathbf{u}, \mathbf{w} \in \mathbf{H}_{0}^{1}, \\
& |b(\mathbf{u}, \mathbf{v}, \mathbf{w})| \leq C\|\mathbf{u}\|^{\frac{1}{2}}\|\mathbf{u}\|_{1}^{\frac{1}{2}}\|\mathbf{v}\|_{1}\|\mathbf{w}\|^{\frac{1}{2}}\|\mathbf{w}\|_{1}^{\frac{1}{2}}, \quad \forall \mathbf{v}, \mathbf{u}, \mathbf{w} \in \mathbf{H}_{0}^{1} ;
\end{aligned}
$$

if $\Omega \in R^{3}$, we have:

$$
|b(\mathbf{u}, \mathbf{v}, \mathbf{w})| \leq C\|\mathbf{u}\|_{1}\|\mathbf{v}\|_{1}^{\frac{1}{2}}\|\mathbf{v}\|_{2}^{\frac{1}{2}}\|\mathbf{w}\|, \quad \forall \mathbf{v} \in \mathbf{H}^{2} \cap \mathbf{H}_{0}^{1}, \mathbf{u}, \mathbf{w} \in \mathbf{H}_{0}^{1},
$$

where the trilinear form $b$ can be replaced with $\bar{b}$. 
We will use the modified nonlinear convection term $\bar{B}(\mathbf{u}, \mathbf{u})$ to replace the original one $B(\mathbf{u}, \mathbf{u})$ in the remaining parts. The Navier-Stokes equations and SRM equations are modified respectively as

$$
\begin{aligned}
& \mathbf{u}_{t}+\bar{B}(\mathbf{u}, \mathbf{u})=\nu \Delta \mathbf{u}-\operatorname{grad} p+\mathbf{f} \\
& \operatorname{div} \mathbf{u}=0, \\
& \left.\mathbf{u}\right|_{\partial \Omega}=\mathbf{0},\left.\mathbf{u}\right|_{t=0}=\mathbf{u}_{0},
\end{aligned}
$$

and

$$
\begin{aligned}
& \left(\mathbf{u}_{s}\right)_{t}+\bar{B}\left(\mathbf{u}_{s}, \mathbf{u}_{s}\right)=\nu \Delta \mathbf{u}_{s}-\operatorname{grad} p_{s}+\mathbf{f}, \\
& \operatorname{div}\left(\left(\mathbf{u}_{s}\right)_{t}+\alpha \mathbf{u}_{s}\right)=\epsilon\left(p_{s-1}-p_{s}\right), \\
& \left.\mathbf{u}_{s}\right|_{\partial \Omega}=\mathbf{0},\left.\mathbf{u}_{s}\right|_{t=0}=\mathbf{u}_{0}
\end{aligned}
$$

We should mention that the systems (1.1)-(1.3) and (2.25)-(2.27) have the same solution.

Now we start to analyze the SRM (2.28)-(2.30). We have the following two lemmas (see [10]), and these lemmas are also true when we replace $B$ by $\bar{B}$.

Lemma 2.3. For the solution $\left\{\mathbf{u}_{s}, p_{s}\right\}$ of (2.28), (2.29), (2.30), we have the following estimates:

$$
\begin{aligned}
& \left\|\mathbf{u}_{s}\right\|_{1}^{2}+\int_{0}^{T}\left(\frac{1}{\epsilon^{2}}\left\|\left(\operatorname{div} \mathbf{u}_{s}\right)_{t}\right\|_{1}^{2}+\frac{1}{\epsilon^{2}}\left\|\operatorname{div} \mathbf{u}_{s}\right\|_{1}^{2}+\left\|\left(\mathbf{u}_{s}\right)_{t}\right\|^{2}+\left\|\mathbf{u}_{s}\right\|_{2}^{2}+\left\|p_{s}\right\|_{1}^{2}\right) d t \\
& \leq C_{1} M_{1, s} e^{C_{1} M_{1, s}^{2}},
\end{aligned}
$$

where $M_{1, s}=\left\|\mathbf{u}_{0}\right\|_{1}^{2}+\int_{0}^{T}\left(\|\mathbf{f}\|^{2}+\left\|p_{s-1}\right\|_{1}^{2}\right) d t$, and $C_{1}$ is a generic constant which does not depend on $\mathbf{f}, \mathbf{u}_{0}$ and $p_{s-1}$.

The second lemma is for a linear auxiliary problem:

$$
\begin{aligned}
\mathbf{w}_{t}+ & \bar{B}(\mathbf{U}, \mathbf{w})+\bar{B}(\mathbf{w}, \mathbf{V})=\nu \Delta \mathbf{w}-\operatorname{grad} p+\mathbf{f}, \\
& (\operatorname{div} \mathbf{w})_{t}+\alpha \operatorname{div} \mathbf{w}=g, \\
& \left.\mathbf{w}\right|_{\partial \Omega}=\mathbf{0},\left.\mathbf{w}\right|_{t=0}=\mathbf{u}_{0},
\end{aligned}
$$

where $\mathbf{U}, \mathbf{V}, g$ and $\mathbf{u}_{0}$ are given functions, $\mathbf{u}_{0}$ satisfies the compatibility conditions (2.14) and $g$ satisfies (2.13).

Lemma 2.4. For the solution of (2.31)-(2.33), if $\mathbf{U}$ and $\mathbf{V}$ satisfy

$$
\|\cdot\|_{1}^{2}+\int_{0}^{T}\|\cdot\|_{2}^{2} d t \leq M_{2}
$$

then we have the following estimate:

$$
\begin{aligned}
& \|\mathbf{w}\|_{1}^{2}+\int_{0}^{T}\left(\|\mathbf{w}\|_{2}^{2}+\left\|\mathbf{w}_{t}\right\|^{2}+\|p\|_{1}^{2}\right) d t \\
& \leq C_{2}\left(1+M_{2}\right) e^{C_{2} M_{2}}\left(\left\|\mathbf{u}_{0}\right\|_{1}^{2}+\int_{0}^{T}\left(\|\mathbf{f}\|^{2}+\|g\|_{1}^{2}\right) d t\right)
\end{aligned}
$$

where $C_{2}$ does not depend on $\mathbf{f}, g$ and $\mathbf{u}_{0}$. 
For Navier-Stokes equations, we have a classical energy bound (see [18]):

$$
\|\mathbf{u}\|_{1}^{2}+\int_{0}^{T}\left(\|\mathbf{u}\|_{2}^{2}+\|p\|_{1}^{2}\right) d t \leq C_{3} M_{3} e^{C_{3} M_{3}^{2}},
$$

where $M_{3}=\left\|\mathbf{u}_{0}\right\|_{1}^{2}+\int_{0}^{T}\|\mathbf{f}\|^{2} d t$ and $C_{3}$ does not depend on $\mathbf{f}, \mathbf{u}_{0}$.

We will prove the convergence results by combining the above lemmas. Let

$$
\begin{aligned}
& C=\max \left\{C_{1}, C_{2}, C_{3}\right\}, M_{4}=2+\left\|\mathbf{u}_{0}\right\|_{1}^{2}+\int_{0}^{T}\left(\|\mathbf{f}\|^{2}+2\|p\|_{1}^{2}\right) d t, \\
& M_{5}=C M_{4} e^{C M_{4}^{2}}, M=C\left(M_{5}+1\right) e^{C M_{5}^{2}}
\end{aligned}
$$

and $\epsilon$ satisfy

$$
4 M \epsilon^{2}\left(1+2 \int_{0}^{T}\left(\|p\|_{1}^{2}+\left\|p_{0}\right\|_{1}^{2}\right) d t\right) \leq 1 .
$$

Theorem 2.5 (Convergence). Let $\mathbf{u}$ and $p$ be the solution of problem (2.25), (2.26), (2.27) and $\mathbf{u}_{s}$ and $p_{s}$ the solution of problem (2.28), (2.29), (2.30). If we choose our initial guess $p_{0}$ to satisfy $\int_{0}^{T}\left\|p_{0}\right\|_{1}^{2} d t \leq 2+2 \int_{0}^{T}\|p\|_{1}^{2} d t$ (e.g. $\left.p_{0}=0\right)$, and $\epsilon$ to satisfy (2.37), then for $s=1,2, \cdots$, we have the following error estimate:

$$
\left\|\mathbf{u}-\mathbf{u}_{s}\right\|_{1}^{2}+\int_{0}^{T}\left(\left\|\mathbf{u}-\mathbf{u}_{s}\right\|_{2}^{2}+\left\|\left(\mathbf{u}-\mathbf{u}_{s}\right)_{t}\right\|^{2}+\left\|p-p_{s}\right\|_{1}^{2}\right) d t \leq\left(4 M \epsilon^{2}\right)^{s} \int_{0}^{T}\left\|p-p_{0}\right\|_{1}^{2} d t .
$$

We have seen most parts of this estimate in [10, where the technique of asymptotic expansion is used in the proof. But here we are going to provide a much simpler proof following an idea in [11. Here we also give clear criteria that $p_{0}$ and $\epsilon$ should satisfy for convergence.

Proof. Subtracting (2.25)-(2.27) (replacing (2.26) by divu $\mathbf{u}_{t}+\alpha \operatorname{div} \mathbf{u}=0$ ) from (2.28)-(2.30) and denoting by $\mathbf{e}_{s}=\mathbf{u}_{s}-\mathbf{u}, h_{s}=p_{s}-p$ for $s=1,2, \cdots$, we have

$$
\begin{aligned}
& \left(\mathbf{e}_{s}\right)_{t}+\bar{B}\left(\mathbf{u}_{s}, \mathbf{e}_{s}\right)+\bar{B}\left(\mathbf{e}_{s}, \mathbf{u}\right)=\nu \Delta \mathbf{e}_{s}-\operatorname{grad}\left(h_{s}\right), \\
& \left(\operatorname{div} \mathbf{e}_{s}\right)_{t}+\alpha \operatorname{div} \mathbf{e}_{s}=\epsilon\left(h_{s-1}-h_{s}\right), \\
& \left.\mathbf{e}_{s}\right|_{\partial \Omega}=\mathbf{0},\left.\mathbf{e}_{s}\right|_{t=0}=\mathbf{0} .
\end{aligned}
$$

Clearly, from estimation (2.36), we have

$$
\|\mathbf{u}\|_{1}^{2}+\int_{0}^{T}\|\mathbf{u}\|_{2}^{2} d t \leq M_{5} .
$$

We will prove the following claims by induction; the convergence is the corollary of claim (2.45).

Claim: For $s=1,2,3, \ldots$, we have

$$
\begin{aligned}
& \left\|\mathbf{u}_{s}\right\|_{1}^{2}+\int_{0}^{T}\left\|\mathbf{u}_{s}\right\| d t \leq M_{5}, \\
& 4 M \epsilon^{2} \int_{0}^{T}\left\|h_{s-1}\right\|_{1}^{2} d t \leq 1, \\
& \left\|\mathbf{e}_{s}\right\|_{1}^{2}+\int_{0}^{T}\left(\left\|\mathbf{e}_{s}\right\|_{2}^{2}+\left\|\left(\mathbf{e}_{s}\right)_{t}\right\|^{2}+\left\|h_{s}\right\|_{1}^{2}\right) d t \leq 4 M \epsilon^{2} \int_{0}^{T}\left\|h_{s-1}\right\|_{1}^{2} d t .
\end{aligned}
$$


Step 1: Case $s=1$.

Since our initial guess $p_{0}$ satisfies $\int_{0}^{T}\left\|p_{0}\right\|_{1}^{2} d t \leq 2+2 \int_{0}^{T}\|p\|_{1}^{2} d t$, hence $M_{1,1} \leq M_{4}$ (see Lemma 2.3 for the definition of $M_{1, s}$ ). Claim (2.43) follows from Lemma 2.3 and the definition of $\epsilon_{0}$ implies claim (2.44). Estimates (2.42) and (2.43) together with Lemma 2.4 imply

$$
\left\|\mathbf{e}_{1}\right\|_{1}^{2}+\int_{0}^{T}\left(\left\|\mathbf{e}_{1}\right\|_{2}^{2}+\left\|\left(\mathbf{e}_{1}\right)_{t}\right\|^{2}+\left\|h_{1}\right\|_{1}^{2}\right) d t \leq M \epsilon^{2} \int_{0}^{T}\left\|h_{0}-h_{1}\right\|_{1}^{2} d t .
$$

Applying the Cauchy inequality, and noticing the choice of $\epsilon$, we have

$$
\begin{gathered}
M \epsilon^{2} \int_{0}^{T}\left\|h_{0}-h_{1}\right\|_{1}^{2} d t \leq 2 M \epsilon^{2} \int_{0}^{T}\left(\left\|h_{0}\right\|_{1}^{2}+\left\|h_{1}\right\|_{1}^{2}\right) d t \\
\quad \leq \frac{1}{2} \int_{0}^{T}\left\|h_{1}\right\|_{1}^{2} d t+2 M \epsilon^{2} \int_{0}^{T}\left\|h_{0}\right\|_{1}^{2} d t .
\end{gathered}
$$

Inequalities (2.46) and (2.47) yield claim (2.45).

Step 2: Assume that claims (2.43) and (2.45) are true for $s=k$ and let $s=k+1$.

Claims (2.45) and (2.44) for $s=k$ imply

$$
\int_{0}^{T}\left\|h_{k}\right\|_{1}^{2} d t \leq 4 M \epsilon^{2} \int_{0}^{T}\left\|h_{k-1}\right\|_{1}^{2} d t \leq 1
$$

This and

$$
\int_{0}^{T}\left\|p_{k}\right\|_{1}^{2} d t=\int_{0}^{T}\left\|h_{k}+p\right\|_{1}^{2} d t \leq 2 \int_{0}^{T}\left(\|p\|_{1}^{2}+\left\|h_{k}\right\|_{1}^{2}\right) d t
$$

give

$M_{1, k+1}=\left\|\mathbf{u}_{0}\right\|_{1}^{2}+\int_{0}^{T}\left(\|\mathbf{f}\|^{2}+\left\|p_{k}\right\|_{1}^{2}\right) d t \leq 2+\left\|\mathbf{u}_{0}\right\|_{1}^{2}+\int_{0}^{T}\left(\|\mathbf{f}\|^{2}+2\|p\|_{1}^{2}\right) d t=M_{4}$.

Claim (2.43) is a corollary of Lemma 2.3 by taking $s=k+1$. The proof of claim (2.45) is similar to the case of $s=1$. (2.42) and (2.43) together with Lemma 2.4 imply

$$
\left\|\mathbf{e}_{k+1}\right\|_{1}^{2}+\int_{0}^{T}\left(\left\|\mathbf{e}_{k+1}\right\|_{2}^{2}+\left\|\left(\mathbf{e}_{k+1}\right)_{t}\right\|^{2}+\left\|h_{k+1}\right\|_{1}^{2}\right) d t \leq M \epsilon^{2} \int_{0}^{T}\left\|h_{k}-h_{k+1}\right\|_{1}^{2} d t .
$$

Applying the Cauchy inequality, and noticing the choice of $\epsilon$, we have

$$
\begin{aligned}
& M \epsilon^{2} \int_{0}^{T}\left\|h_{k}-h_{k+1}\right\|_{1}^{2} d t \leq 2 M \epsilon^{2} \int_{0}^{T}\left(\left\|h_{k}\right\|_{1}^{2}+\left\|h_{k+1}\right\|_{1}^{2}\right) d t \\
& \leq \frac{1}{2} \int_{0}^{T}\left\|h_{k+1}\right\|_{1}^{2} d t+2 M \epsilon^{2} \int_{0}^{T}\left\|h_{k}\right\|_{1}^{2} d t .
\end{aligned}
$$

The above two inequalities yield claim (2.45). Claim (2.45) immediately implies

$$
4 M \epsilon^{2} \int_{0}^{T}\left\|h_{k+1}\right\|_{1}^{2} d t \leq 4 M \epsilon^{2}\left(4 M \epsilon^{2} \int_{0}^{T}\left\|h_{k}\right\|_{1}^{2} d t\right) \leq 4 M \epsilon^{2} \leq 1 .
$$

So the claim (2.44) is also true for $s=k+1$.

Remark 2.1. As a corollary, we can obtain $\left\|p_{s-1}-p_{s}\right\|_{L^{2}\left(H^{1}\right)}=O\left(\epsilon^{s-1}\right)$ from inequality (2.38); hence the divergence of the velocity is of $O\left(\epsilon^{s}\right)$. 
Remark 2.2. From Theorem 2.5, the choice of $\epsilon$ should satisfy (2.37). In practice, it is not necessarily very small. A good approximation can be ensured by iterations as long as $\epsilon=o(1)$ (see numerical results in [10, 12]).

\section{Existence of THE STRONG SOlution}

When we apply SRM to Navier-Stokes equations, we need to solve equations (2.28)-(2.30) at each iteration step. By eliminating $p_{s}$ from equations (2.28)-(2.30), omitting the index $s$ and denoting the right-hand side as a new function $\mathbf{f}$, we obtain equations which are similar to (1.7), (1.8):

$$
\begin{aligned}
& \mathbf{u}_{t}-\frac{1}{\epsilon} \operatorname{grad} \operatorname{div}\left(\mathbf{u}_{t}+\alpha \mathbf{u}\right)-\nu \Delta \mathbf{u}+\bar{B}(\mathbf{u}, \mathbf{u})=\mathbf{f}, \\
& \left.\mathbf{u}\right|_{\partial \Omega}=0,\left.\mathbf{u}\right|_{t=0}=\mathbf{u}_{0},
\end{aligned}
$$

where $\operatorname{div} \mathbf{u}_{0}=0$. We will establish the existence and uniqueness of the solution of equation (3.1), (3.2) in this section. Following standard ideas we need to obtain energy estimates of a semi-discretized problem and then pass to the limit of the discrete solution. There are two ways to construct the discrete solution, i.e. temporally discrete or spatially discrete solution. If we use the spatially discrete solution, it is difficult to obtain enough energy or regularity estimates (since we do not have enough boundary conditions to ensure the integration by parts). So we turn to time discretization (see also [13] for using the temporally discrete solution). We first establish the energy estimations for the time discrete scheme, then let the time step $\triangle t$ approach 0 . The limit function is the strong solution of problem (3.1), (3.2).

To take a semi-discrete scheme in time, we first use a semi-implicit scheme, i.e. an explicit-implicit scheme for the nonlinear convention term and an implicit scheme for the remaining. The system reads:

$$
\begin{aligned}
& \frac{\mathbf{u}^{n+1}-\mathbf{u}^{n}}{\triangle t}-\frac{1}{\epsilon} \operatorname{grad} \operatorname{div}\left(\frac{\mathbf{u}^{n+1}-\mathbf{u}^{n}}{\triangle t}+\alpha \mathbf{u}^{n+1}\right) \\
& -\nu \triangle \mathbf{u}^{n+1}+\bar{B}\left(\mathbf{u}^{n}, \mathbf{u}^{n+1}\right)=\mathbf{f}^{n+1}
\end{aligned}
$$

with the homogeneous Dirichlet boundary condition, where $\mathbf{u}^{0}=\mathbf{u}_{0}, \mathbf{f}^{n+1}=$ $\mathbf{f}\left(\mathbf{x}, t_{n+1}\right)$ and $t_{n}=n \triangle t$. Most of our results are obtained for this semi-implicit scheme. But in practice, we are also interested in a fully explicit treatment of the nonlinear convection term (thus the stiffness matrix will not change in time). The corresponding time-discrete scheme reads:

$$
\frac{\mathbf{u}^{n+1}-\mathbf{u}^{n}}{\triangle t}-\frac{1}{\epsilon} \operatorname{grad} \operatorname{div}\left(\frac{\mathbf{u}^{n+1}-\mathbf{u}^{n}}{\triangle t}+\alpha \mathbf{u}^{n+1}\right)-\nu \triangle \mathbf{u}^{n+1}+\bar{B}\left(\mathbf{u}^{n}, \mathbf{u}^{n}\right)=\mathbf{f}^{n+1}
$$

satisfying the same boundary and initial conditions. If we only consider a short time solution ( $\Omega$ can be in either $2 \mathrm{D}$ or $3 \mathrm{D}$ ), we have a similar estimation for this scheme. We will also consider a finite time solution in a remark.

Clearly, equations (3.3) and (3.4) are linear second order elliptic equations for any fixed $n$. The existence and uniqueness of the solutions are standard. Moreover, we have stronger energy estimations for both equations.

Lemma 3.1. Assume that the time interval is $[0, T]$. Let $N=\frac{T}{\triangle t}$, and define $A \mathbf{u}^{n+1}=-\frac{1}{\epsilon} \operatorname{grad} \operatorname{div}\left(\frac{\mathbf{u}^{n+1}-\mathbf{u}^{n}}{\Delta t}+\alpha \mathbf{u}^{n+1}\right)-\nu \triangle \mathbf{u}^{n+1}, n=0,1,2,3 \ldots$ and $\operatorname{div} \mathbf{u}^{0}=0$. 
Then there exists a constant $\epsilon_{0}$ which does not depend on $\mathbf{u}^{n}$, such that when $\epsilon \leq \epsilon_{0}$, we have

$$
\begin{aligned}
& \triangle t \sum_{1}^{N}\left(\left\|\mathbf{u}^{n}\right\|_{2}^{2}+\frac{1}{\epsilon^{2}}\left\|\operatorname{grad} \operatorname{div} \mathbf{u}^{n}\right\|^{2}+\frac{1}{\epsilon^{2}}\left\|\operatorname{grad} \operatorname{div} \frac{\mathbf{u}^{n}-\mathbf{u}^{n-1}}{\triangle t}\right\|^{2}\right) \leq C \triangle t \sum_{1}^{N}\left\|A \mathbf{u}^{n}\right\|^{2}, \\
& \sup _{1 \leq n \leq N}\left(\left\|\mathbf{u}^{n}\right\|_{2}+\frac{1}{\epsilon}\left\|\operatorname{grad} \operatorname{div} \mathbf{u}^{n}\right\|+\frac{1}{\epsilon}\left\|\operatorname{grad} \operatorname{div} \frac{\mathbf{u}^{n}-\mathbf{u}^{n-1}}{\triangle t}\right\|\right) \leq C \sup _{1 \leq n \leq N}\left\|A \mathbf{u}^{n}\right\|,
\end{aligned}
$$

where the constant $C$ does not depend on the choice of $\epsilon$ and $\mathbf{u}^{n}$.

Proof. Define $\mathbf{w}^{n}=A \mathbf{u}^{n}, p^{n}=-\frac{1}{\epsilon} \operatorname{div}\left(\frac{\mathbf{u}^{n}-\mathbf{u}^{n-1}}{\Delta t}+\alpha \mathbf{u}^{n}\right)$. Then

$$
\begin{array}{r}
-\nu \triangle \mathbf{u}^{n}+\operatorname{grad} p^{n}=\mathbf{w}^{n}, \\
\operatorname{div}\left(\frac{\mathbf{u}^{n}-\mathbf{u}^{n-1}}{\triangle t}+\alpha \mathbf{u}^{n}\right)=-\epsilon p^{n} .
\end{array}
$$

Solving equation (3.6), and defining $g^{n}=\operatorname{div} \mathbf{u}^{n}$, we have

$$
\begin{array}{r}
(1+\alpha \triangle t) g^{n}-g^{n-1}=-\epsilon \triangle t p^{n} \\
\Rightarrow\left\|\operatorname{grad} g^{n}\right\| \leq\left\|\operatorname{grad} g^{0}\right\|+\epsilon \triangle t \sum_{i=0}^{n}\left\|\operatorname{grad} p^{i}\right\| .
\end{array}
$$

Since $\mathbf{u}^{0}$ is divergence free, the first term on the right-hand side of above equation is 0 . Then we apply the Cauchy-Schwarz inequality,

$$
\begin{aligned}
\left\|\operatorname{grad} g^{n}\right\|^{2} & \leq \epsilon^{2} \triangle t^{2}\left\|\sum_{i=1}^{n} \operatorname{grad} p^{i}\right\|^{2} \\
& \leq \epsilon^{2} \triangle t^{2} n \sum_{1}^{n}\left\|\operatorname{grad} p^{i}\right\|^{2} \\
& \leq \epsilon^{2} T \triangle t \sum_{1}^{n}\left\|\operatorname{grad} p^{i}\right\|^{2} .
\end{aligned}
$$

Using the inequality (1.9) for the inhomogeneous Stokes equations (see [18]), we have

$$
\left\|\mathbf{u}^{n}\right\|_{2}^{2}+\left\|\operatorname{grad} p^{n}\right\|^{2} \leq C_{1}\left(\left\|\mathbf{w}^{n}\right\|^{2}+\left\|\operatorname{grad} g^{n}\right\|^{2}\right)
$$

Defining the constant $\epsilon_{0}=\min \left(\frac{1}{\sqrt{2 C_{1} T^{2}}}, \frac{1}{2 C_{1} T}\right)$, taking the summation from 1 to $n$ in inequality (3.10) and using inequality (3.9), we have

$$
\triangle t \sum_{1}^{n}\left(\left\|\mathbf{u}^{i}\right\|_{2}^{2}+\left\|\operatorname{grad} p^{i}\right\|^{2}\right) \leq C_{2} \triangle t \sum_{1}^{n}\left\|\mathbf{w}^{i}\right\|^{2} \quad \text { for any } \epsilon \leq \epsilon_{0} .
$$

From the definition of $p^{i}$, we obtain the first conclusion in the lemma. To obtain the uniform estimation, we take sup in inequality (3.8), that is,

$$
\begin{aligned}
\sup _{1 \leq n \leq N}\left\|\operatorname{grad} g^{n}\right\| & \leq \sup _{1 \leq n \leq N} \epsilon \triangle t \sum_{i=0}^{n}\left\|\operatorname{grad} p^{i}\right\| \\
& \leq \sup _{1 \leq n \leq N} \epsilon \triangle t N\left\|\operatorname{grad} p^{n}\right\| .
\end{aligned}
$$

Then taking sup at inequality (3.10) and choosing $\epsilon \leq \epsilon_{0}$, we complete the second part of this lemma. 
Lemma 3.2. For equation (3.3), if we assume that $\triangle t \sum_{0}^{N}\left\|\mathbf{f}^{n}\right\|_{-1}^{2}$ is bounded, $\mathbf{u}^{0} \in \mathbf{L}^{2}$ and $\operatorname{div} \mathbf{u}^{0}=0$, we have the following estimation:

$$
\left\|\mathbf{u}^{n}\right\|^{2}+\frac{1}{\epsilon}\left\|\operatorname{div} \mathbf{u}^{n}\right\|^{2}+\triangle t \sum_{1}^{n}\left\|\mathbf{u}^{i}\right\|_{1}^{2} \leq C .
$$

Proof. Multiplying by $\mathbf{u}^{n+1}$ on both sides of equations (3.3), we have:

$$
\begin{aligned}
\frac{1}{2 \triangle t} & \left(\left\|\mathbf{u}^{n+1}\right\|^{2}-\left\|\mathbf{u}^{n}\right\|^{2}+\left\|\mathbf{u}^{n+1}-\mathbf{u}^{n}\right\|^{2}\right) \\
& +\frac{1}{2 \triangle t \epsilon}\left(\left\|\operatorname{div} \mathbf{u}^{n+1}\right\|^{2}-\left\|\operatorname{div} \mathbf{u}^{n}\right\|^{2}+\left\|\operatorname{div}\left(\mathbf{u}^{n+1}-\mathbf{u}^{n}\right)\right\|^{2}\right) \\
& +\frac{\alpha}{\epsilon}\left\|\operatorname{div} \mathbf{u}^{n+1}\right\|^{2} \nu\left\|\operatorname{grad} \mathbf{u}^{n+1}\right\|^{2}+\bar{b}\left(\mathbf{u}^{n}, \mathbf{u}^{n+1}, \mathbf{u}^{n+1}\right)=\left(\mathbf{f}^{n+1}, \mathbf{u}^{n+1}\right) .
\end{aligned}
$$

Since $\bar{b}\left(\mathbf{u}^{n}, \mathbf{u}^{n+1}, \mathbf{u}^{n+1}\right)=0$ and $\left(\mathbf{f}^{n+1}, \mathbf{u}^{n+1}\right) \leq \frac{\nu}{2}\left\|\operatorname{gradu}^{n+1}\right\|^{2}+C_{1}\left\|\mathbf{f}^{n+1}\right\|_{-1}^{2}$, we can then define $y^{n}=\left\|\mathbf{u}^{n}\right\|^{2}+\frac{1+\alpha \triangle t}{\epsilon}\left\|\operatorname{div} \mathbf{u}^{n}\right\|^{2}$ to obtain the inequality

$$
y^{n+1}-y^{n}+\triangle t\left\|\operatorname{gradu}^{n+1}\right\|^{2} \leq C_{2} \triangle t\left\|\mathbf{f}^{n+1}\right\|_{-1}^{2} .
$$

Taking the summation from 0 to $n$ in the above inequality yields

$$
y^{n}-y^{0}+\triangle t \sum_{1}^{n}\left\|\operatorname{gradu}^{i}\right\|^{2} \leq C_{2} \triangle t \sum_{1}^{n}\left\|\mathbf{f}^{i}\right\|_{-1}^{2} .
$$

Since $y^{0}=\left\|\mathbf{u}^{0}\right\|^{2}$ is bounded, we conclude the lemma.

With more regularity assumptions for $\mathbf{f}$ and $\mathbf{u}^{0}$, we can have a higher regularity estimation.

Lemma 3.3. For equation (3.3), if we assume that $\triangle t \sum_{0}^{N}\left\|\mathbf{f}^{n}\right\|^{2}$ is bounded, $\mathbf{u}^{0} \in$ $\mathbf{H}_{0}^{1} \cap \mathbf{H}^{2}, \operatorname{div} \mathbf{u}^{0}=0$ and $\epsilon \leq \epsilon_{0}$, we have the following estimation:

$$
\begin{gathered}
\left\|\mathbf{u}^{n+1}\right\|_{1}^{2}+\triangle t \sum_{0}^{n}\left\{\frac{1}{\epsilon^{2}}\left\|\operatorname{grad} \operatorname{div} \frac{\mathbf{u}^{i+1}-\mathbf{u}^{i}}{\triangle t}\right\|^{2}+\frac{1}{\epsilon^{2}}\left\|\operatorname{grad} \operatorname{div} \mathbf{u}^{i+1}\right\|^{2}\right. \\
\left.+\left\|\triangle \mathbf{u}^{i+1}\right\|^{2}+\left\|\frac{\mathbf{u}^{i+1}-\mathbf{u}^{i}}{\triangle t}\right\|^{2}\right\} \leq C .
\end{gathered}
$$

Proof. In Lemma 3.2, we have proved that $\sup \left\|\mathbf{u}^{n}\right\|$ and $\triangle t \sum_{1}^{n}\left\|\mathbf{u}^{i}\right\|_{1}^{2}$ are bounded, which will be used in the proof without so mentioning. Multiplying by $\frac{\mathbf{u}^{n+1}-\mathbf{u}^{n}}{\Delta t}$ on both sides of equation (3.3), we have

$$
\begin{aligned}
& \left\|\frac{\mathbf{u}^{n+1}-\mathbf{u}^{n}}{\triangle t}\right\|^{2}+\frac{\nu}{2 \triangle t}\left(\left\|\operatorname{gradu}^{n+1}\right\|^{2}-\left\|\operatorname{grad} \mathbf{u}^{n}\right\|^{2}+\left\|\operatorname{grad}\left(\mathbf{u}^{n+1}-\mathbf{u}^{n}\right)\right\|^{2}\right) \\
& +\frac{1}{\epsilon}\left\|\operatorname{div}\left(\frac{\mathbf{u}^{n+1}-\mathbf{u}^{n}}{\triangle t}\right)\right\|^{2}+\frac{\alpha}{2 \epsilon \triangle t}\left(\left\|\operatorname{div} \mathbf{u}^{n+1}\right\|^{2}-\left\|\operatorname{div} \mathbf{u}^{n}\right\|^{2}+\left\|\operatorname{div}\left(\mathbf{u}^{n+1}-\mathbf{u}^{n}\right)\right\|^{2}\right) \\
& +\bar{b}\left(\mathbf{u}^{n}, \mathbf{u}^{n+1}, \frac{\mathbf{u}^{n+1}-\mathbf{u}^{n}}{\triangle t}\right)=\left(\mathbf{f}^{n+1}, \frac{\mathbf{u}^{n+1}-\mathbf{u}^{n}}{\triangle t}\right) .
\end{aligned}
$$

Since

$$
\begin{aligned}
\left|\bar{b}\left(\mathbf{u}^{n}, \mathbf{u}^{n+1}, \frac{\mathbf{u}^{n+1}-\mathbf{u}^{n}}{\triangle t}\right)\right| & \leq C_{1}\left\|\mathbf{u}^{n}\right\|^{\frac{1}{2}}\left\|\mathbf{u}^{n}\right\|_{1}^{\frac{1}{2}}\left\|\mathbf{u}^{n+1}\right\|_{1}^{\frac{1}{2}}\left\|\mathbf{u}^{n+1}\right\|_{2}^{\frac{1}{2}}\left\|\frac{\mathbf{u}^{n+1}-\mathbf{u}^{n}}{\triangle t}\right\| \\
& \leq \frac{1}{4}\left\|\frac{\mathbf{u}^{n+1}-\mathbf{u}^{n}}{\triangle t}\right\|^{2}+C_{1}^{2}\left\|\mathbf{u}^{n}\right\|\left\|\mathbf{u}^{n}\right\|_{1}\left\|\mathbf{u}^{n+1}\right\|_{1}\left\|\mathbf{u}^{n+1}\right\|_{2} \\
& \leq \frac{1}{4}\left\|\frac{\mathbf{u}^{n+1}-\mathbf{u}^{n}}{\triangle t}\right\|^{2}+\delta\left\|\mathbf{u}^{n+1}\right\|_{2}^{2}+\frac{C_{2}}{\delta}\left\|\mathbf{u}^{n}\right\|_{1}^{2}\left\|\mathbf{u}^{n+1}\right\|_{1}^{2}
\end{aligned}
$$


and

$$
\left(\mathbf{f}^{n+1}, \frac{\mathbf{u}^{n+1}-\mathbf{u}^{n}}{\triangle t}\right) \leq \frac{1}{4}\left\|\frac{\mathbf{u}^{n+1}-\mathbf{u}^{n}}{\triangle t}\right\|^{2}+\left\|\mathbf{f}^{n+1}\right\|^{2},
$$

we have

$$
\begin{aligned}
& \left\|\frac{\mathbf{u}^{n+1}-\mathbf{u}^{n}}{\triangle t}\right\|^{2}+\frac{1}{\epsilon \triangle t}\left(\left\|\operatorname{div} \mathbf{u}^{n+1}\right\|^{2}-\left\|\operatorname{div} \mathbf{u}^{n}\right\|^{2}\right) \\
& \quad+\frac{1}{\triangle t}\left(\left\|\operatorname{gradu}^{n+1}\right\|^{2}-\left\|\operatorname{gradu}^{n}\right\|^{2}\right) \\
& \leq C_{3}\left\|\mathbf{f}^{n+1}\right\|^{2}+\delta\left\|\mathbf{u}^{n+1}\right\|_{2}^{2}+C_{\delta}\left\|\mathbf{u}^{n}\right\|_{1}^{2}\left\|\mathbf{u}^{n+1}\right\|_{1}^{2} .
\end{aligned}
$$

If we rewrite the equation (3.3) as

$$
\begin{aligned}
& -\nu \triangle \mathbf{u}^{n+1}+\operatorname{grad} p^{n+1}=\mathbf{f}^{n+1}-\frac{\mathbf{u}^{n+1}-\mathbf{u}^{n}}{\triangle t}-\bar{B}\left(\mathbf{u}^{n}, \mathbf{u}^{n+1}\right), \\
& \operatorname{div} \mathbf{u}^{n+1}=g^{n+1}, \quad(1+\alpha \triangle t) g^{n+1}-g^{n}=-\epsilon \triangle t p^{n+1},
\end{aligned}
$$

by applying estimation (1.9) for the nonhomogeneous Stokes equation, and using Young's inequality and Sobolev's inequality, we have

$$
\begin{aligned}
& \left\|\mathbf{u}^{n+1}\right\|_{2}^{2}+\left\|\operatorname{grad} p^{n+1}\right\| \leq C_{0}\left(\left\|\mathbf{f}^{n+1}\right\|^{2}+\left\|\frac{\mathbf{u}^{n+1}-\mathbf{u}^{n}}{\Delta t}\right\|^{2}\right. \\
& \left.+\left\|\operatorname{grad} g^{n+1}\right\|^{2}+\delta\left\|\mathbf{u}^{n+1}\right\|_{2}^{2}+C_{\delta}\left\|\mathbf{u}^{n}\right\|_{1}^{2}\left\|\mathbf{u}^{n+1}\right\|_{1}^{2}\right) .
\end{aligned}
$$

Then (3.14) $+\frac{1}{2 C_{0}} \times(3.15)$ implies

$$
\begin{aligned}
& \frac{1}{2}\left\|\frac{\mathbf{u}^{n+1}-\mathbf{u}^{n}}{\triangle t}\right\|^{2}+\frac{1}{2 C_{0}}\left(\left\|\mathbf{u}^{n+1}\right\|_{2}^{2}+\left\|\operatorname{grad} p^{n+1}\right\|^{2}\right)+\frac{1}{\epsilon \triangle t}\left(\left\|\operatorname{div} \mathbf{u}^{n+1}\right\|^{2}-\left\|\operatorname{div} \mathbf{u}^{n}\right\|^{2}\right) \\
& +\frac{1}{\triangle t}\left(\left\|\operatorname{grad} \mathbf{u}^{n+1}\right\|^{2}-\left\|\operatorname{grad} \mathbf{u}^{n}\right\|^{2}\right) \leq\left(C_{3}+1\right)\left\|\mathbf{f}^{n+1}\right\|^{2}+\left\|\operatorname{grad} g^{n+1}\right\|^{2} \\
& +\delta\left\|\mathbf{u}^{n+1}\right\|_{2}^{2}+C_{\delta}\left\|\mathbf{u}^{n}\right\|_{1}^{2}\left\|\mathbf{u}^{n+1}\right\|_{1}^{2} .
\end{aligned}
$$

Multiplying by $\operatorname{grad} g^{n+1}$ on both sides of the recursive equation $(1+\alpha \triangle t) \operatorname{grad} g^{n+1}$ $-\operatorname{grad} g^{n}=-\epsilon \triangle \operatorname{tgrad} p^{n+1}$, we have

$$
\begin{aligned}
& \frac{1}{2}\left(\left\|\operatorname{grad} g^{n+1}\right\|^{2}-\left\|\operatorname{grad} g^{n}\right\|^{2}+\left\|\operatorname{grad}\left(g^{n+1}-g^{n}\right)\right\|^{2}\right)+\alpha \triangle t\left\|\operatorname{grad} g^{n+1}\right\|^{2} \\
& =-\epsilon \triangle t\left(\operatorname{grad} p^{n+1}, \operatorname{grad} g^{n+1}\right) \leq \frac{\epsilon^{2} \triangle t}{2 \alpha}\left\|\operatorname{grad} p^{n+1}\right\|^{2}+\frac{\alpha \triangle t}{2}\left\|\operatorname{grad} g^{n+1}\right\|^{2} .
\end{aligned}
$$

Therefore,

$$
\frac{1}{\triangle t}\left(\left\|\operatorname{grad} g^{n+1}\right\|^{2}-\left\|\operatorname{grad} g^{n}\right\|^{2}\right)+\alpha\left\|\operatorname{grad} g^{n+1}\right\|^{2} \leq \frac{\epsilon^{2}}{\alpha}\left\|\operatorname{grad} p^{n+1}\right\|^{2} .
$$

Denoting $y^{n}=\frac{1}{\epsilon^{2}}\left\|\operatorname{grad} g^{n}\right\|^{2}, z^{n}=\frac{1}{\epsilon}\left\|\operatorname{div} \mathbf{u}^{n}\right\|^{2}+\left\|\operatorname{grad} \mathbf{u}^{n}\right\|^{2}$, then

$$
\begin{aligned}
& \frac{1}{2}\left\|\frac{\mathbf{u}^{n+1}-\mathbf{u}^{n}}{\triangle t}\right\|^{2}+\frac{1}{2 C_{0}}\left\|\mathbf{u}^{n+1}\right\|_{2}^{2}+\frac{\alpha^{2}}{2 C_{0}} y^{n+1}+\frac{1}{\triangle t} \frac{\alpha}{2 C_{0}}\left(y^{n+1}-y^{n}\right)+\frac{1}{\triangle t}\left(z^{n+1}-z^{n}\right) \\
& \leq\left(C_{3}+1\right)\left\|\mathbf{f}^{n+1}\right\|^{2}+\epsilon^{2} y^{n+1}+\delta\left\|\mathbf{u}^{n+1}\right\|_{2}^{2}+C_{\delta}\left\|\mathbf{u}^{n}\right\|_{1}^{2} z^{n+1} .
\end{aligned}
$$

If $\epsilon$ is less than some critical value $\epsilon_{0}$ (e.g. $\frac{\alpha}{\sqrt{2 C_{0}}}$ ), we can choose a properly small $\delta$ such that

$$
\left\|\frac{\mathbf{u}^{n+1}-\mathbf{u}^{n}}{\triangle t}\right\|^{2}+\left\|\mathbf{u}^{n+1}\right\|_{2}^{2}+\frac{1}{\triangle t}\left(y^{n+1}+z^{n+1}-y^{n}-z^{n}\right) \leq C_{4}\left(\left\|\mathbf{f}^{n+1}\right\|^{2}+\left\|\mathbf{u}^{n}\right\|_{1}^{2} z^{n+1}\right) .
$$


Applying the discrete Gronwall's inequality yields

$$
\left\|\mathbf{u}^{n+1}\right\|_{1}^{2}+\triangle t \sum_{0}^{n}\left\{\frac{1}{\epsilon^{2}}\left\|\operatorname{grad} \operatorname{div} \mathbf{u}^{i+1}\right\|^{2}+\left\|\triangle \mathbf{u}^{i+1}\right\|^{2}+\left\|\frac{\mathbf{u}^{i+1}-\mathbf{u}^{i}}{\Delta t}\right\|^{2}\right\} \leq C .
$$

The last term $\triangle t \sum_{0}^{n} \frac{1}{\epsilon^{2}}\left\|\operatorname{grad} \operatorname{div} \frac{\mathbf{u}^{i+1}-\mathbf{u}^{i}}{\Delta t}\right\|^{2}$ can be obtained from the identity

$$
\frac{1}{\epsilon} \frac{g^{n+1}-g^{n}}{\triangle t}=-p^{n+1}-\frac{\alpha}{\epsilon} g^{n+1},
$$

where $\triangle t \sum_{1}^{n}\left\|\nabla p^{i}\right\|^{2}$ is bounded by (3.15).

Now with the estimation (3.13), we can pass to the limit and then prove the existence of the strong solution. Define the strong solution to equation (3.1)-(3.2) as follows.

Problem 3.1. For $\mathbf{f} \in L^{2}\left(\mathbf{L}^{2}\right)$ and $\mathbf{u}_{0} \in \mathbf{H}_{0}^{1} \cap \mathbf{H}^{2}$ with divergence free, find $\mathbf{u}$ to satisfy $\mathbf{u} \in L^{2}\left(\mathbf{H}_{0}^{1} \cap \mathbf{H}^{2}\right), \mathbf{u}_{t} \in L^{2}\left(\mathbf{L}^{2}\right)$, divu $\mathbf{u}_{t} \in L^{2}\left(\mathbf{H}^{1}\right)$, and

$$
\begin{aligned}
& \mathbf{u}_{t}-\frac{1}{\epsilon} \operatorname{grad} \operatorname{div}\left(\mathbf{u}_{t}+\alpha \mathbf{u}\right)-\nu \Delta \mathbf{u}+\bar{B}(\mathbf{u}, \mathbf{u})=\mathbf{f}, \\
& \left.\mathbf{u}\right|_{t=0}=\mathbf{u}_{0} .
\end{aligned}
$$

Theorem 3.4. There exists a unique solution $\mathbf{u}$ to problem 3.1 .

Proof. We introduce the time semi-discrete solution which depends on the time step $\triangle t$, and define $u^{n}$ to be a function of spatial variables satisfying the time semi-discrete equations (3.3). Then define $\mathbf{u}_{\triangle t}$ and $\mathbf{U}_{\triangle t}$ to be a piecewise constant or linear interpolation of $u^{n}$, respectively,

$$
\begin{array}{ll}
\mathbf{u}_{\triangle t}(\cdot, t)=\mathbf{u}^{n+1}, & t \in[n \triangle t,(n+1) \triangle t), \\
\mathbf{U}_{\triangle t}(\cdot, t)=\mathbf{u}^{n}+\frac{t-n \triangle t}{\triangle t}\left(\mathbf{u}^{n+1}-\mathbf{u}^{n}\right), & t \in[n \triangle t,(n+1) \triangle t) .
\end{array}
$$

Then we can rewrite equation (3.3) as

$$
\frac{\partial}{\partial t} \mathbf{U}_{\triangle t}-\frac{1}{\epsilon} \operatorname{grad} \operatorname{div}\left(\frac{\partial}{\partial t} \mathbf{U}_{\triangle t}+\mathbf{u}_{\triangle t}\right)-\nu \triangle \mathbf{u}_{\triangle t}+\bar{B}\left(\mathbf{u}_{\triangle t}(\cdot, t-\triangle t), \mathbf{u}_{\triangle t}\right)=\mathbf{f}_{\triangle t},
$$

where $\mathbf{f}_{\triangle t}$ is a constant interpolation of $\mathbf{f}^{n+1}$ in the time step. Clearly, $\mathbf{u}_{\triangle t}$ is bounded in $L^{2}\left(\mathbf{H}_{0}^{1} \cap \mathbf{H}^{2}\right)$ and $\mathbf{U}_{\triangle t}$ is bounded in $L^{2}\left(\mathbf{H}_{0}^{1} \cap \mathbf{H}^{2}\right) \cap H^{1}\left(\mathbf{L}^{2}\right)$. Passing to the limit of subsequence $\triangle t_{j}$, we have

$$
\begin{aligned}
& \mathbf{u}_{\triangle t_{j}} \stackrel{w}{\longrightarrow} \mathbf{u}^{*} \in L^{2}\left(\mathbf{H}_{0}^{1} \cap \mathbf{H}^{2}\right), \\
& \mathbf{U}_{\triangle t_{j}} \stackrel{w}{\sim} \mathbf{u} \in L^{2}\left(\mathbf{H}_{0}^{1} \cap \mathbf{H}^{2}\right) \cap H^{1}\left(\mathbf{L}^{2}\right) .
\end{aligned}
$$

Define $Q=[0, T] \times \Omega$, and

$$
L^{2}(Q)=L^{2}\left(L^{2}(\Omega)\right), \quad H^{1}(Q)=\left\{u: u \in L^{2}(Q), \operatorname{grad} u \in L^{2}(Q), u_{t} \in L^{2}(Q)\right\} .
$$

Then we have $L^{2}\left(\mathbf{H}_{0}^{1} \cap \mathbf{H}^{2}\right) \cap H^{1}\left(\mathbf{L}^{2}\right) \hookrightarrow \mathbf{H}^{1}(Q) \hookrightarrow \mathbf{L}^{2}(Q)$, where the second embedding is compact (see [5]); hence

$$
\mathbf{U}_{\triangle t_{j}} \rightarrow \mathbf{u} \in L^{2}\left(\mathbf{L}^{2}\right) \text {. }
$$

Then we estimate the difference between $\mathbf{u}_{\triangle t}$ and $\mathbf{U}_{\triangle t}$. By the definition of $\mathbf{u}_{\triangle t}$ and $\mathbf{U}_{\triangle t}$,

$$
\left\|\mathbf{u}_{\triangle t}(\cdot, t)-\mathbf{U}_{\triangle t}(\cdot, t)\right\| \leq\left\|\mathbf{u}^{n+1}-\mathbf{u}^{n}\right\|, \quad t \in[n \triangle t,(n+1) \triangle t) .
$$


Hence,

$$
\int_{0}^{T}\left\|\mathbf{u}_{\triangle t}-\mathbf{U}_{\triangle t}\right\|^{2} d t \leq \triangle t \sum_{0}^{N-1}\left\|\mathbf{u}^{n+1}-\mathbf{u}^{n}\right\|^{2}
$$

Then by Lemma 3.3 we get $\left\|\mathbf{u}_{\triangle t}-\mathbf{U}_{\triangle t}\right\|_{L^{2}(Q)}^{2} \leq C \triangle t^{2}$. Hence, $\mathbf{u}^{*}$ and $\mathbf{u}$ are the same element in $L^{2}(Q)$. Thus we also obtain that

$$
\mathbf{u}_{\triangle t_{j}} \rightarrow \mathbf{u} \in L^{2}\left(\mathbf{L}^{2}\right) .
$$

The next step is to check that $\mathbf{u}$ is indeed a strong solution. Choosing a test function $\mathbf{v}$ to be smooth enough in $Q$ and taking the inner product with $\mathbf{v}$ for equation (3.19), we can pass to the limit weakly in $L^{2}(Q)$ for all terms in (3.19) except the nonlinear term. Consider the nonlinear term $\bar{B}\left(\mathbf{u}_{\triangle t}(\cdot, t-\triangle t), \mathbf{u}_{\triangle t}\right)$. Since grad $\mathbf{u}_{\triangle t}$ converges to grad $\mathbf{u}$ weakly and $\mathbf{u}_{\triangle t}$ converges to $\mathbf{u}$ strongly in $L^{2}(Q)$, we conclude that $\bar{B}\left(\mathbf{u}_{\triangle t}(\cdot, t-\triangle t), \mathbf{u}_{\triangle t}\right)$ converges to $\bar{B}(\mathbf{u}, \mathbf{u})$ in the sense of distribution on $Q$ (see [18]). After passing to the limit, we obtain $\mathbf{u}$ satisfying equation (3.16).

Then we need to verify that $\mathbf{u}$ satisfies the initial condition $\mathbf{u}(0)=\mathbf{u}_{0}$. There is a standard embedding result, $L^{2}\left(\mathbf{H}_{0}^{1} \cap \mathbf{H}^{2}\right) \cap H^{1}\left(\mathbf{L}^{2}\right) \hookrightarrow C\left(\mathbf{H}^{1}\right)$ (see [5]), and then the map $\mathbf{u} \rightarrow \mathbf{u}(0)$ is continuous from $C\left(\mathbf{H}^{1}\right)$ to $\mathbf{H}^{1}$. From above, the initial condition is satisfied automatically.

The last step is to check the uniqueness of the solution. Assume that we have two solutions $\mathbf{u}$ and $\mathbf{v}$. Define $\mathbf{w}=\mathbf{u}-\mathbf{v}$. Then we have the equation of $\mathbf{w}$ :

$$
\mathbf{w}_{t}-\frac{1}{\epsilon} \operatorname{grad} \operatorname{div}\left(\mathbf{w}_{t}+\alpha \mathbf{w}\right)-\nu \Delta \mathbf{w}+\bar{B}(\mathbf{u}, \mathbf{w})+\bar{B}(\mathbf{w}, \mathbf{v})=\mathbf{0}
$$

with homogeneous Dirichlet boundary condition and zero initial condition. Multiplying the above equation by $\mathbf{w}_{t}$ and applying Sobolev's and Young's inequalities, we have

$$
\frac{d}{d t}\left(\frac{1}{\epsilon}\|\operatorname{div} \mathbf{w}\|^{2}+\|\operatorname{grad} \mathbf{w}\|^{2}\right) \leq C\left(\|\mathbf{u}\|_{2}^{2}+\|\mathbf{v}\|_{2}^{2}\right)\|\operatorname{grad} \mathbf{w}\|^{2} .
$$

Since $\mathbf{u}$ and $\mathbf{v}$ are in $L^{2}\left(\mathbf{H}^{2}\right)$ and from $\mathbf{w}(0)=\mathbf{0}$ and Gronwall's inequality we have $\operatorname{grad} \mathbf{w}=0$, hence the uniqueness of solution follows.

As we mentioned before, if we only look for a short time solution, the fully explicit scheme for the nonlinear convection term may be analyzed accordingly as well. Moreover, the argument works for the 3D case. The following lemma is similar to Lemma 3.3 .

Lemma 3.5. Assume that $\triangle t \sum_{0}^{N}\left\|\mathbf{f}^{n}\right\|^{2}$ is bounded, $\mathbf{u}^{0} \in \mathbf{H}_{0}^{1} \cap \mathbf{H}^{2}$, divu $\mathbf{u}^{0}=0$, and $\epsilon$ is less than a positive constant $\epsilon_{0}$. Then there exists a positive constant $T_{0}$, whenever $0<n \triangle t \leq T^{*}=\min \left(T, T_{0}\right)$, and the solution to equation (3.4) satisfies

$$
\begin{aligned}
\left\|\mathbf{u}^{n+1}\right\|_{1}^{2}+\Delta & t \sum_{0}^{n}\left(\frac{1}{\epsilon^{2}}\left\|\operatorname{grad} \operatorname{div} \frac{\mathbf{u}^{i+1}-\mathbf{u}^{i}}{\triangle t}\right\|^{2}\right. \\
& \left.+\frac{1}{\epsilon^{2}}\left\|\operatorname{grad} \operatorname{div} \mathbf{u}^{i+1}\right\|^{2}+\left\|\triangle \mathbf{u}^{i+1}\right\|^{2}+\left\|\frac{\mathbf{u}^{i+1}-\mathbf{u}^{i}}{\triangle t}\right\|^{2}\right) \leq C .
\end{aligned}
$$


Proof. The proof will be slightly different from Lemma 3.3 since we do not have an a priori estimation for the solution of scheme (3.4). Multiplying by $\frac{\mathbf{u}^{n+1}-\mathbf{u}^{n}}{\Delta t}$ on both sides of equation (3.4), similarly to Lemma 3.3, but noticing

$$
\begin{aligned}
\left|\bar{b}\left(\mathbf{u}^{n}, \mathbf{u}^{n}, \frac{\mathbf{u}^{n+1}-\mathbf{u}^{n}}{\triangle t}\right)\right| & \leq C_{1}\left\|\mathbf{u}^{n}\right\|_{1}^{\frac{3}{2}}\left\|\mathbf{u}^{n}\right\|_{2}^{\frac{1}{2}}\left\|\frac{\mathbf{u}^{n+1}-\mathbf{u}^{n}}{\triangle t}\right\| \\
& \leq \frac{1}{4}\left\|\frac{\mathbf{u}^{n+1}-\mathbf{u}^{n}}{\triangle t}\right\|^{2}+\delta\left\|\mathbf{u}^{n}\right\|_{2}^{2}+C_{\delta}\left\|\mathbf{u}^{n}\right\|_{1}^{6},
\end{aligned}
$$

we have

$$
\begin{aligned}
& \left\|\frac{\mathbf{u}^{n+1}-\mathbf{u}^{n}}{\triangle t}\right\|^{2}+\frac{1}{\epsilon \triangle t}\left(\left\|\operatorname{div} \mathbf{u}^{n+1}\right\|^{2}-\left\|\operatorname{div} \mathbf{u}^{n}\right\|^{2}\right) \\
& \quad+\frac{1}{\triangle t}\left(\left\|\operatorname{gradu}^{n+1}\right\|^{2}-\left\|\operatorname{gradu}^{n}\right\|^{2}\right) \leq C_{2}\left\|\mathbf{f}^{n+1}\right\|^{2}+\delta\left\|\mathbf{u}^{n}\right\|_{2}^{2}+C_{\delta}\left\|\mathbf{u}^{n}\right\|_{1}^{6} .
\end{aligned}
$$

Then we rewrite the equation (3.4) as

$$
\begin{aligned}
& -\nu \triangle \mathbf{u}^{n+1}+\operatorname{grad} p^{n+1}=\mathbf{f}^{n+1}-\frac{\mathbf{u}^{n+1}-\mathbf{u}^{n}}{\triangle t}-\bar{B}\left(\mathbf{u}^{n}, \mathbf{u}^{n}\right), \\
& \operatorname{div} \mathbf{u}^{n+1}=g^{n+1}, \quad(1+\alpha \triangle t) g^{n+1}-g^{n}=-\epsilon \triangle t p^{n+1} .
\end{aligned}
$$

By applying estimation (1.9) for the nonhomogeneous Stokes equation, Young's and Sobolev's inequalities, we obtain

$$
\begin{gathered}
\left\|\mathbf{u}^{n+1}\right\|_{2}^{2}+\left\|\operatorname{grad} p^{n+1}\right\| \leq C_{0}\left(\left\|\mathbf{f}^{n+1}\right\|^{2}+\left\|\frac{\mathbf{u}^{n+1}-\mathbf{u}^{n}}{\triangle t}\right\|^{2}\right. \\
\left.+\left\|\operatorname{grad} g^{n+1}\right\|^{2}+\delta\left\|\mathbf{u}^{n}\right\|_{2}^{2}+C_{\delta}\left\|\mathbf{u}^{n}\right\|_{1}^{6}\right) .
\end{gathered}
$$

Then $(\underline{3.24})+\frac{1}{2 C_{0}} \times(3.25)$ implies

$$
\begin{gathered}
\frac{1}{2}\left\|\frac{\mathbf{u}^{n+1}-\mathbf{u}^{n}}{\triangle t}\right\|^{2}+\frac{1}{2 C_{0}}\left(\left\|\mathbf{u}^{n+1}\right\|_{2}^{2}+\left\|\operatorname{grad} p^{n+1}\right\|^{2}\right) \\
+\frac{1}{\epsilon \triangle t}\left(\left\|\operatorname{div} \mathbf{u}^{n+1}\right\|^{2}-\left\|\operatorname{div} \mathbf{u}^{n}\right\|^{2}\right)+\frac{1}{\triangle t}\left(\left\|\operatorname{grad} \mathbf{u}^{n+1}\right\|^{2}-\left\|\operatorname{grad} \mathbf{u}^{n}\right\|^{2}\right) \\
\leq\left(C_{3}+1\right)\left\|\mathbf{f}^{n+1}\right\|^{2}+\left\|\operatorname{grad} g^{n+1}\right\|^{2}+\delta\left\|\mathbf{u}^{n}\right\|_{2}^{2}+C_{\delta}\left\|\mathbf{u}^{n}\right\|_{1}^{6} .
\end{gathered}
$$

Multiplying by $\operatorname{grad} g^{n+1}$ on both sides of the recursive equation for $\operatorname{grad} g^{n}$,

$$
(1+\alpha \triangle t) \operatorname{grad} g^{n+1}-\operatorname{grad} g^{n}=-\epsilon \triangle t \operatorname{grad} p^{n+1},
$$

we have

$$
\begin{aligned}
& \frac{1}{2}\left(\left\|\operatorname{grad} g^{n+1}\right\|^{2}-\left\|\operatorname{grad} g^{n}\right\|^{2}+\left\|\operatorname{grad}\left(g^{n+1}-g^{n}\right)\right\|^{2}\right)+\alpha \triangle t\left\|\operatorname{grad} g^{n+1}\right\|^{2} \\
& =-\epsilon \triangle t\left(\operatorname{grad} p^{n+1}, \operatorname{grad} g^{n+1}\right) \leq \frac{\epsilon^{2} \triangle t}{2 \alpha}\left\|\operatorname{grad} p^{n+1}\right\|^{2}+\frac{\alpha \triangle t}{2}\left\|\operatorname{grad} g^{n+1}\right\|^{2} ;
\end{aligned}
$$

hence

$$
\frac{1}{\triangle t}\left(\left\|\operatorname{grad} g^{n+1}\right\|^{2}-\left\|\operatorname{grad} g^{n}\right\|^{2}\right)+\alpha\left\|\operatorname{grad} g^{n+1}\right\|^{2} \leq \frac{\epsilon^{2}}{\alpha}\left\|\operatorname{grad} p^{n+1}\right\|^{2} .
$$


Define $y^{n}=\frac{1}{\epsilon^{2}}\left\|\operatorname{grad} g^{n}\right\|^{2}, z^{n}=\frac{1}{\epsilon}\left\|\operatorname{div} \mathbf{u}^{n}\right\|^{2}+\left\|\operatorname{grad} \mathbf{u}^{n}\right\|^{2}$. Then

$$
\begin{aligned}
& \frac{1}{2}\left\|\frac{\mathbf{u}^{n+1}-\mathbf{u}^{n}}{\triangle t}\right\|^{2}+\frac{1}{2 C_{0}}\left\|\mathbf{u}^{n+1}\right\|_{2}^{2}+\frac{\alpha^{2}}{2 C_{0}} y^{n+1}+\frac{1}{\triangle t} \frac{\alpha}{2 C_{0}}\left(y^{n+1}-y^{n}\right)+\frac{1}{\triangle t}\left(z^{n+1}-z^{n}\right) \\
& \leq\left(C_{3}+1\right)\left\|\mathbf{f}^{n+1}\right\|^{2}+\epsilon^{2} y^{n+1}+\delta\left\|\mathbf{u}^{n}\right\|_{2}^{2}+C_{\delta}\left(z^{n}\right)^{3} .
\end{aligned}
$$

If $\epsilon$ is less than some critical value $\epsilon_{0}$ (e.g. $\frac{\alpha}{\sqrt{2 C_{0}}}$ ), we can choose a properly small $\delta$ (e.g. $\left.\delta=\frac{1}{4 C_{0}}\right)$, and denote $d^{n}=y^{n}+z^{n}+\triangle t\left\|\mathbf{u}^{n}\right\|_{2}^{2}$; hence

$$
\left\|\frac{\mathbf{u}^{n+1}-\mathbf{u}^{n}}{\triangle t}\right\|^{2}+\left\|\mathbf{u}^{n+1}\right\|_{2}^{2}+\frac{1}{\triangle t}\left(d^{n+1}-d^{n}\right) \leq C_{4}\left(\left\|\mathbf{f}^{n+1}\right\|^{2}+\left(z^{n}\right)^{3}\right) .
$$

Summing for $n$ yields

$$
\triangle t \sum_{0}^{n}\left(\left\|\frac{\mathbf{u}^{i+1}-\mathbf{u}^{i}}{\triangle t}\right\|^{2}+\left\|\mathbf{u}^{i+1}\right\|_{2}^{2}\right)+d^{n+1} \leq d^{0}+C_{4} \triangle t \sum_{0}^{n}\left(\left\|\mathbf{f}^{i+1}\right\|^{2}+\left(d^{n}\right)^{3}\right) .
$$

Denoting by $w^{n}=d^{0}+C_{4} \triangle t \sum_{0}^{N}\left\|\mathbf{f}^{i}\right\|^{2}+C_{4} \triangle t \sum_{0}^{n}\left(d^{n}\right)^{3}$, and noticing $d^{n+1} \leq w^{n}$ from the above inequality, we then have

$$
w^{n+1}-w^{n}=C_{4} \triangle t\left(d^{n+1}\right)^{3} \leq C_{4} \triangle t\left(w^{n}\right)^{3} .
$$

Now consider a concave function $F(x)=-\frac{1}{2 x^{2}}$. Clearly $F(x)$ is negative and monotonically increasing in $(0, \infty)$, and $F^{\prime}(x)=\frac{1}{x^{3}}$. By the convexity of $F$,

$$
F\left(w^{n+1}\right)-F\left(w^{n}\right) \leq F^{\prime}\left(w^{n}\right)\left(w^{n+1}-w^{n}\right)=\frac{w^{n+1}-w^{n}}{\left(w^{n}\right)^{3}} \leq C_{4} \triangle t .
$$

Hence $F\left(w^{n}\right) \leq F\left(w^{0}\right)+n C_{4} \triangle t$. Since $F\left(w^{0}\right)$ is negative, we let $T_{0}$ be the critical time that satisfies $C^{*}=F\left(w^{0}\right)+C_{4} T_{0}<0$ and $T^{*}=\min \left(T, T_{0}\right)$. As long as $n \triangle t \leq T^{*}$, we have $w^{n} \leq F^{-1}\left(C^{*}\right)$ and hence the estimation

$$
\left\|\mathbf{u}^{n+1}\right\|_{1}^{2}+\triangle t \sum_{0}^{n}\left(\frac{1}{\epsilon^{2}}\left\|\operatorname{grad} \operatorname{div} \mathbf{u}^{i+1}\right\|^{2}+\left\|\triangle \mathbf{u}^{i+1}\right\|^{2}+\left\|\frac{\mathbf{u}^{i+1}-\mathbf{u}^{i}}{\triangle t}\right\|^{2}\right) \leq C
$$

The last term $\triangle t \sum_{0}^{n} \frac{1}{\epsilon^{2}}\left\|\operatorname{grad} \operatorname{div} \frac{\mathbf{u}^{i+1}-\mathbf{u}^{i}}{\Delta t}\right\|^{2} \leq C$ can be obtained by the same argument as in Lemma 3.3 .

Remark 3.1. Lemma 3.5 states the stability of the fully explicit treatment of the nonlinear convection term. With this energy estimation, we can prove the existence of a strong solution in a short time for equation (3.1), (3.2) when $\Omega \in R^{3}$. The proof will be exactly the same as that of Lemma 3.5 just replacing $T$ by $T^{*}$.

\section{ERror estimation of the time Discretization}

In this section, we will estimate the error between the SRM solution and the semi-implicit time discrete solution. Hence, combining it with Theorem 2.5 we can obtain an error estimate between the solution of the semi-implicit scheme and the solution of the Navier-Stokes equations (1.1)-(1.2). Subtracting equation (3.1) from equation (3.3), denoting the error function by $\mathbf{e}^{n}=\mathbf{u}^{n}-\mathbf{u}\left(t_{n}\right)$, where $t_{n}=n \triangle t$, we obtain the equation that the error $\mathbf{e}^{n}$ satisfies:

$$
\begin{aligned}
& \frac{\mathbf{e}^{n+1}-\mathbf{e}^{n}}{\triangle t}-\frac{1}{\epsilon} \operatorname{grad} \operatorname{div}\left(\frac{\mathbf{e}^{n+1}-\mathbf{e}^{n}}{\triangle t}+\alpha \mathbf{e}^{n+1}\right)-\nu \triangle \mathbf{e}^{n+1} \\
& +\bar{B}\left(\mathbf{u}^{n}, \mathbf{u}^{n+1}\right)-\bar{B}\left(\mathbf{u}\left(t_{n+1}\right), \mathbf{u}\left(t_{n+1}\right)\right)=\mathbf{r}^{n+1},
\end{aligned}
$$


where the remainder term $\mathbf{r}^{n+1}=\frac{1}{\Delta t} \int_{t_{n}}^{t_{n+1}}\left(\eta-t_{n}\right)\left[\mathbf{u}_{t t}(\eta)-\frac{1}{\epsilon} \operatorname{grad} \operatorname{div} \mathbf{u}_{t t}(\eta)\right] d \eta$. Since $\triangle t=t_{n+1}-t_{n}$, we have

$$
\begin{aligned}
\left\|\mathbf{r}^{n+1}\right\|^{2} & \leq \frac{1}{\triangle t^{2}} \int_{t_{n}}^{t_{n+1}}\left|\eta-t_{n}\right|^{2} d \eta \int_{t_{n}}^{t_{n+1}}\left\|\mathbf{u}_{t t}(\eta)-\frac{1}{\epsilon} \operatorname{graddiv} \mathbf{u}_{t t}(\eta)\right\|^{2} d \eta \\
& =\frac{\triangle t}{3} \int_{t_{n}}^{t_{n+1}}\left\|\mathbf{u}_{t t}(\eta)-\frac{1}{\epsilon} \operatorname{grad} \operatorname{div} \mathbf{u}_{t t}(\eta)\right\|^{2} d \eta
\end{aligned}
$$

Hence we need an energy estimation for $\int_{0}^{T}\left\|\mathbf{u}_{t t}(\eta)-\frac{1}{\epsilon} \operatorname{grad} \operatorname{div} \mathbf{u}_{t t}(\eta)\right\|^{2} d \eta$ to control the truncation error.

\subsection{A priori estimation.}

Lemma 4.1. Define an operator $A \mathbf{u}=-\frac{1}{\epsilon} \operatorname{grad} \operatorname{div}\left(\mathbf{u}_{t}+\alpha \mathbf{u}\right)-\nu \Delta \mathbf{u}$, where the time interval is $[0, T]$. Then there exists a constant $\epsilon_{0}$ independent of $\mathbf{u}$ and when $\epsilon \leq \epsilon_{0}$, we have

$$
\begin{aligned}
& \int_{0}^{t} \frac{1}{\epsilon^{2}}\left\|\operatorname{grad} \operatorname{div} \mathbf{u}_{t}\right\|^{2}+\frac{1}{\epsilon^{2}}\|\operatorname{graddiv} \mathbf{u}\|^{2}+\|\Delta \mathbf{u}\|^{2} d t \leqq C\left(\|\operatorname{graddiv} \mathbf{u}(0)\|^{2}+\int_{0}^{t}\|A \mathbf{u}\|^{2} d t\right), \\
& \sup _{t \in[0, T]}\left(\frac{1}{\epsilon}\left\|\operatorname{grad} \operatorname{div} \mathbf{u}_{t}\right\|+\frac{1}{\epsilon}\|\operatorname{grad} \operatorname{div} \mathbf{u}\|+\|\Delta \mathbf{u}\|\right) \leqq C\left(\|\operatorname{graddiv} \mathbf{u}(0)\|+\sup _{t \in[0, T]}\|A \mathbf{u}\|\right),
\end{aligned}
$$

where the constant $C$ does not depend on $\epsilon$ and $\mathbf{u}$.

Proof. The proof is similar to the discrete case, Lemma 3.1. Let $\mathbf{w}=A \mathbf{u}, p=$ $-\frac{1}{\epsilon} \operatorname{div}\left(\mathbf{u}_{t}+\alpha \mathbf{u}\right)$ and $g=\operatorname{div} \mathbf{u}$. Firstly we solve for $g$ from the ODE

$$
g_{t}+\alpha g=-\epsilon p
$$

with initial condition $g(0)=\operatorname{div} \mathbf{u}(0)$. The solution is

$$
g(t)=e^{-\alpha t} g(0)-\epsilon \int_{0}^{t} p(s) e^{\alpha(s-t)} d s .
$$

Now $(\mathbf{u}, p)$ satisfies the inhomogeneous Stokes equations

$$
\begin{array}{r}
-\triangle \mathbf{u}+\operatorname{grad} p=\mathbf{w}, \\
\operatorname{div} \mathbf{u}=g .
\end{array}
$$

Then using the estimation (1.9), we have

$$
\|\mathbf{u}\|_{2}+\|\operatorname{grad} p\| \leq C_{0}(\|\mathbf{w}\|+\|\operatorname{grad} g\|) .
$$

Hence

$$
\|\mathbf{u}\|_{2}+\|\operatorname{grad} p\| \leqq C_{1}\left(\|\operatorname{grad} \operatorname{div} \mathbf{u}(0)\|+\|\mathbf{w}\|+\epsilon \int_{0}^{t}\|\operatorname{grad} p(s)\| d s\right)
$$

Choosing $\epsilon$ to be small enough $\left(\epsilon \leq \epsilon_{0}=\frac{1}{2 C_{1} T}\right)$, we can obtain

$$
\sup _{t \in[0, T]}\left(\|\mathbf{u}\|_{2}+\|\operatorname{grad} p\|\right) \leqq C\left(\|\operatorname{grad} \operatorname{div} \mathbf{u}(0)\|+\sup _{t \in[0, T]}\|\mathbf{w}\|\right)
$$

and

$$
\int_{0}^{t}\|\mathbf{u}\|_{2}^{2}+\|\operatorname{grad} p\|^{2} d s \leqq C\left(\|\operatorname{grad} \operatorname{div} \mathbf{u}(0)\|^{2}+\int_{0}^{t}\|\mathbf{w}\|^{2} d s\right) .
$$

From the above inequalities and the definition of $p$, we can conclude the lemma.

The following lemmas give a higher regularity estimate for the solution of equation (3.1)- (3.2). 
Lemma 4.2. Let $\mathbf{f} \in L^{\infty}\left(\mathbf{L}^{2}\right), \mathbf{f}_{t} \in L^{2}\left(\mathbf{H}^{-1}\right), \mathbf{u}_{0} \in \mathbf{H}^{2} \cap \mathbf{H}_{0}^{1}$, $\operatorname{div} \mathbf{u}_{0}=0$ and $\epsilon \leq \epsilon_{0}$. Then we have:

$$
\sup _{t \in[0, T]}\left(\left\|\mathbf{u}_{t}\right\|+\|\mathbf{u}\|_{2}+\frac{1}{\epsilon}\left\|\operatorname{grad} \operatorname{div} \mathbf{u}_{t}\right\|+\frac{1}{\epsilon}\|\operatorname{grad} \operatorname{div} \mathbf{u}\|\right) \leq C .
$$

Proof. Multiplying by $\mathbf{u}_{t}$ on both sides of equation (3.1), we have

$$
\left\|\mathbf{u}_{t}\right\|^{2}+\frac{1}{\epsilon}\left\|\operatorname{div} \mathbf{u}_{t}\right\|^{2}-\left(\frac{\alpha}{\epsilon} \operatorname{grad} \operatorname{div} \mathbf{u}, \mathbf{u}_{t}\right)-\left(\nu \Delta \mathbf{u}, \mathbf{u}_{t}\right)+\bar{b}\left(\mathbf{u}, \mathbf{u}, \mathbf{u}_{t}\right)=\left(\mathbf{f}, \mathbf{u}_{t}\right)
$$

Notice that

$$
\begin{aligned}
\left|\left(\frac{\alpha}{\epsilon} \operatorname{grad} \operatorname{div} \mathbf{u}, \mathbf{u}_{t}\right)\right| & \leq\left\|\frac{\alpha}{\epsilon} \operatorname{grad} \operatorname{div} \mathbf{u}\right\|^{2}+\frac{1}{4}\left\|\mathbf{u}_{t}\right\|^{2}, \\
\left|\left(\nu \Delta \mathbf{u}, \mathbf{u}_{t}\right)\right| & \leq\|\nu \Delta \mathbf{u}\|^{2}+\frac{1}{4}\left\|\mathbf{u}_{t}\right\|^{2} \\
\left|\bar{b}\left(\mathbf{u}, \mathbf{u}, \mathbf{u}_{t}\right)\right| & \leq C_{1}\|\mathbf{u}\|_{1}\|\mathbf{u}\|_{2}\left\|\mathbf{u}_{t}\right\| \\
& \leq C_{2}\|\mathbf{u}\|_{2}^{2}+\frac{1}{8}\left\|\mathbf{u}_{t}\right\|^{2}
\end{aligned}
$$

and

Hence

$$
\left(\mathbf{f}, \mathbf{u}_{t}\right) \leq\|\mathbf{f}\|^{2}+\frac{1}{4}\left\|\mathbf{u}_{t}\right\|^{2}
$$

$$
\left\|\mathbf{u}_{t}\right\|^{2}+\frac{1}{\epsilon}\left\|\operatorname{div} \mathbf{u}_{t}\right\|^{2} \leq C_{3}\left(\left\|\frac{1}{\epsilon} \operatorname{grad} \operatorname{div} \mathbf{u}\right\|^{2}+\|\mathbf{u}\|_{2}^{2}+\|\mathbf{f}\|^{2}\right) .
$$

Choosing the time $t=0$ in the above inequality, and noting that $\operatorname{div} \mathbf{u}_{0}=0$, $\mathbf{u}_{0} \in \mathbf{H}^{2}$ and $\mathbf{f} \in L^{\infty}\left(\mathbf{L}^{2}\right)$, we have

$$
\left\|\mathbf{u}_{t}(0)\right\|^{2}+\frac{1}{\epsilon}\left\|\operatorname{div} \mathbf{u}_{t}(0)\right\|^{2} \leq C_{4} .
$$

Then taking the derivative of equation (3.1) with respect to $t$, we have

$$
\mathbf{u}_{t t}-\frac{1}{\epsilon} \operatorname{grad} \operatorname{div}\left(\mathbf{u}_{t t}+\alpha \mathbf{u}_{t}\right)-\nu \Delta \mathbf{u}_{t}+\bar{B}\left(\mathbf{u}, \mathbf{u}_{t}\right)+\bar{B}\left(\mathbf{u}_{t}, \mathbf{u}\right)=\mathbf{f}_{t} .
$$

Multiplying by $\mathbf{u}_{t}$ on both sides of the above equation yields

$$
\frac{1}{2} \frac{d}{d t}\left\|\mathbf{u}_{t}\right\|^{2}+\frac{1}{2 \epsilon} \frac{d}{d t}\left\|\operatorname{div} \mathbf{u}_{t}\right\|^{2}+\frac{\alpha}{\epsilon}\left\|\operatorname{div} \mathbf{u}_{t}\right\|^{2}+\nu\left\|\operatorname{gradu} \mathbf{u}_{t}\right\|^{2}+\bar{b}\left(\mathbf{u}_{t}, \mathbf{u}, \mathbf{u}_{t}\right)+\bar{b}\left(\mathbf{u}, \mathbf{u}_{t}, \mathbf{u}_{t}\right)=\left\langle\mathbf{f}_{t}, \mathbf{u}_{t}\right\rangle .
$$

Using

$$
\begin{aligned}
\left|b\left(\mathbf{u}_{t}, \mathbf{u}, \mathbf{u}_{t}\right)\right| & \leq C_{1}\|\mathbf{u}\|_{2}\left\|\mathbf{u}_{t}\right\|_{1}\left\|\mathbf{u}_{t}\right\| \\
& \leq C_{5}\|\mathbf{u}\|_{2}^{2}\left\|\mathbf{u}_{t}\right\|^{2}+\frac{\nu}{4}\left\|\operatorname{grad}_{t}\right\|^{2} \\
b\left(\mathbf{u}, \mathbf{u}_{t}, \mathbf{u}_{t}\right) & =0 \\
\left\langle\mathbf{f}_{t}, \mathbf{u}_{t}\right\rangle & \leq \frac{1}{\nu}\left\|\mathbf{f}_{t}\right\|_{-1}^{2}+\frac{\nu}{4}\left\|\operatorname{grad} \mathbf{u}_{t}\right\|^{2}
\end{aligned}
$$

we have

$$
\frac{d}{d t}\left\|\mathbf{u}_{t}\right\|^{2}+\frac{1}{\epsilon} \frac{d}{d t}\left\|\operatorname{div} \mathbf{u}_{t}\right\|^{2}+\frac{1}{\epsilon}\left\|\operatorname{div} \mathbf{u}_{t}\right\|^{2}+\left\|\operatorname{grad} \mathbf{u}_{t}\right\|^{2} \leq C_{6}\left(\|\mathbf{u}\|_{2}^{2}\left\|\mathbf{u}_{t}\right\|^{2}+\left\|\mathbf{f}_{t}\right\|_{-1}^{2}\right) .
$$

Since we have already had $\mathbf{u} \in L^{2}\left(\mathbf{H}^{2}\right)$ (Lemma 2.3), applying Gronwall's inequality, we obtain

$$
\left\|\mathbf{u}_{t}\right\|^{2}+\frac{1}{\epsilon}\left\|\operatorname{div} \mathbf{u}_{t}\right\|^{2}+\int_{0}^{t}\left(\frac{\alpha}{\epsilon}\left\|\operatorname{div} \mathbf{u}_{t}\right\|^{2}+\left\|\mathbf{u}_{t}\right\|_{1}^{2}\right) d t \leq C_{7}\left(\int_{0}^{t}\left\|\mathbf{f}_{t}\right\|_{-1}^{2} d t+\left\|\mathbf{u}_{t}(0)\right\|^{2}+\frac{1}{\epsilon}\left\|\operatorname{div} \mathbf{u}_{t}(0)\right\|^{2}\right) .
$$


Applying the inequality (4.8) to control the right-hand side of the above inequality, we have the estimation for $\left\|\mathbf{u}_{t}\right\|^{2}$, i.e.

$$
\left\|\mathbf{u}_{t}\right\|^{2}+\frac{1}{\epsilon}\left\|\operatorname{div} \mathbf{u}_{t}\right\|^{2}+\int_{0}^{t}\left(\frac{1}{\epsilon}\left\|\operatorname{div} \mathbf{u}_{t}\right\|^{2}+\left\|\mathbf{u}_{t}\right\|_{1}^{2}\right) d t \leq C_{8} .
$$

Then multiplying by $A \mathbf{u}$ on both sides of equation (3.1), we have

$$
\left(\mathbf{u}_{t}, A \mathbf{u}\right)+\|A \mathbf{u}\|^{2}+\bar{b}(\mathbf{u}, \mathbf{u}, A \mathbf{u})=(\mathbf{f}, A \mathbf{u}) .
$$

Since

$$
\begin{aligned}
\left|\left(\mathbf{u}_{t}, A \mathbf{u}\right)\right| & \leq\left\|\mathbf{u}_{t}\right\|^{2}+\frac{1}{4}\|A \mathbf{u}\|^{2}, \\
(\mathbf{f}, A \mathbf{u}) & \leq\|\mathbf{f}\|^{2}+\frac{1}{4}\|A \mathbf{u}\|^{2}, \\
|b(\mathbf{u}, \mathbf{u}, A \mathbf{u})| & \leq C_{1}\|\mathbf{u}\|_{1}^{\frac{3}{2}}\|\mathbf{u}\|_{2}^{\frac{1}{2}}\|A \mathbf{u}\| \\
& \leq \frac{C_{9}}{4 \delta}\|\mathbf{u}\|_{1}^{6}+\delta\|\mathbf{u}\|_{2}^{2}+\frac{1}{4}\|A \mathbf{u}\|^{2},
\end{aligned}
$$

we have

$$
\frac{1}{4}\|A \mathbf{u}\|^{2} \leq\left\|\mathbf{u}_{t}\right\|^{2}+\frac{C_{9}}{4 \delta}\|\mathbf{u}\|_{1}^{6}+\delta\|\mathbf{u}\|_{2}^{2}+\|\mathbf{f}\|^{2} .
$$

Taking sup for both sides of the above inequality, using Lemma 4.1 choosing a small $\delta\left(\leq \frac{1}{8 C}\right)$ and noticing that $\|\mathbf{u}\|_{1},\|\mathbf{f}\|$ and $\left\|\mathbf{u}_{t}\right\|$ are bounded, we have

$$
\sup _{t \in[0, T]}\|A \mathbf{u}\| \leq C \text {. }
$$

Finally applying Lemma 4.1 and noting $\operatorname{div} \mathbf{u}_{0}=0$, we obtain the estimation for all terms in the lemma.

Lemma 4.3. Assume all conditions of Lemma 4.2. Moreover, $\mathbf{f}_{t} \in L^{2}\left(\mathbf{L}^{2}\right), \mathbf{g}=$ $\mathbf{f}(0)+\nu \Delta \mathbf{u}_{0}-\bar{B}\left(\mathbf{u}_{0}, \mathbf{u}_{0}\right) \in \mathbf{H}^{1}$ and $\operatorname{divg}=0$. Then we have

$$
\left\|\mathbf{u}_{t}\right\|_{1}^{2}+\int_{0}^{T} \frac{1}{\epsilon^{2}}\left\|\operatorname{grad} \operatorname{div} \mathbf{u}_{t t}\right\|^{2}+\frac{1}{\epsilon^{2}}\left\|\operatorname{grad} \operatorname{div} \mathbf{u}_{t}\right\|^{2}+\left\|\mathbf{u}_{t}\right\|_{2}^{2}+\left\|\mathbf{u}_{t t}\right\|^{2} \leqq C .
$$

Proof. Defining $\mathbf{h}=\mathbf{u}_{t}(0)$ and letting $t=0$ in equation (3.1), we have

$$
\mathbf{h}-\frac{1}{\epsilon} \operatorname{grad} \operatorname{div} \mathbf{h}=\mathbf{g} .
$$

From the regularity result for the second order elliptic equation, we have

$$
\forall v \in C_{0}^{\infty}(\Omega), \quad \exists w \in C_{0}^{\infty}(\Omega), \quad \text { s.t. } w-\frac{1}{\epsilon} \Delta w=v .
$$

Since $\mathbf{h} \in \mathbf{H}(\operatorname{div}) \triangleq\left\{\mathbf{u}: \mathbf{u} \in \mathbf{L}^{2}, \operatorname{div} \mathbf{u} \in L^{2}\right\}$ from (4.8), we have

$$
\begin{aligned}
& \int_{\Omega}(\operatorname{divh}) v d x=\int_{\Omega}(\operatorname{divh})\left(w-\frac{1}{\epsilon} \Delta w\right) d x=\int_{\Omega}(\operatorname{divh}) w d x-\int_{\Omega}(\operatorname{divh}) \frac{1}{\epsilon} \operatorname{divgrad} w d x \\
& =-\int_{\Omega} \mathbf{h} \cdot \operatorname{grad} w d x+\int_{\Omega} \frac{1}{\epsilon} \operatorname{grad} \operatorname{grvh} \cdot \mathbf{g r a d} w d x=-\int_{\Omega}\left(\mathbf{h}-\frac{1}{\epsilon} \operatorname{grad} \operatorname{divh}\right) \cdot \operatorname{grad} w d x \\
& =-\int_{\Omega} \mathbf{g} \cdot \mathbf{g r a d} w d x=\int_{\Omega}(\operatorname{divg}) w d x=0 .
\end{aligned}
$$

Hence $\operatorname{div} \mathbf{h}=0$ in the distribution sense, and $\mathbf{h} \in \mathbf{H}(\operatorname{div})$ implies $\operatorname{div} \mathbf{h}=0$. Then $\mathbf{h}=\mathbf{g} \in \mathbf{H}^{1}$; hence $\left\|\mathbf{u}_{t}(0)\right\|_{1}$ is bounded. Multiplying by $A \mathbf{u}_{t}$ on both sides of equation (4.9), we have

$$
\left(\mathbf{u}_{t t}, A \mathbf{u}_{t}\right)+\left\|A \mathbf{u}_{t}\right\|^{2}+\bar{b}\left(\mathbf{u}_{t}, \mathbf{u}, A \mathbf{u}_{t}\right)+\bar{b}\left(\mathbf{u}, \mathbf{u}_{t}, A \mathbf{u}_{t}\right)=\left(\mathbf{f}_{t}, A \mathbf{u}_{t}\right) .
$$


Using

$$
\begin{aligned}
\left(\mathbf{u}_{t t}, A \mathbf{u}_{t}\right)=\frac{1}{\epsilon}\left\|\operatorname{div} \mathbf{u}_{t t}\right\|^{2} & +\frac{\alpha}{2 \epsilon} \frac{d}{d t}\left\|\operatorname{div} \mathbf{u}_{t}\right\|^{2}+\frac{\nu}{2} \frac{d}{d t}\left\|\operatorname{grad} \mathbf{u}_{t}\right\|^{2} \\
\left|\bar{b}\left(\mathbf{u}_{t}, \mathbf{u}, A \mathbf{u}_{t}\right)\right| & \leq C_{1}\left\|\mathbf{u}_{t}\right\|_{1}\|\mathbf{u}\|_{2}\left\|A \mathbf{u}_{t}\right\| \\
& \leq \frac{1}{4}\left\|A \mathbf{u}_{t}\right\|^{2}+C_{2}\left\|\mathbf{u}_{t}\right\|_{1}^{2} \\
\left|\bar{b}\left(\mathbf{u}, \mathbf{u}_{t}, A \mathbf{u}_{t}\right)\right| & \leq \frac{1}{4}\left\|A \mathbf{u}_{t}\right\|^{2}+C_{2}\left\|\mathbf{u}_{t}\right\|_{1}^{2} \\
\left(\mathbf{f}_{t}, A \mathbf{u}_{t}\right) & \leq \frac{1}{4}\left\|A \mathbf{u}_{t}\right\|^{2}+\left\|\mathbf{f}_{t}\right\|^{2}
\end{aligned}
$$

we have

$$
\frac{1}{\epsilon}\left\|\operatorname{div} \mathbf{u}_{t t}\right\|^{2}+\frac{1}{\epsilon} \frac{d}{d t}\left\|\operatorname{div} \mathbf{u}_{t}\right\|^{2}+\frac{d}{d t}\left\|\mathbf{u}_{t}\right\|_{1}^{2}+\left\|A \mathbf{u}_{t}\right\|^{2} \leq C_{3}\left(\left\|\mathbf{u}_{t}\right\|_{1}^{2}+\left\|\mathbf{f}_{t}\right\|^{2}\right) .
$$

Applying Gronwall's inequality, we obtain

$$
\left\|\mathbf{u}_{t}\right\|_{1}^{2}+\int_{0}^{T}\left\|A \mathbf{u}_{t}\right\|^{2} \leq C_{4}
$$

Multiplying by $\mathbf{u}_{t t}$ on both sides of equation (4.9), we have

$$
\left\|\mathbf{u}_{t t}\right\|^{2}+\left(A \mathbf{u}_{t}, \mathbf{u}_{t t}\right)+\bar{b}\left(\mathbf{u}_{t}, \mathbf{u}, \mathbf{u}_{t t}\right)+\bar{b}\left(\mathbf{u}, \mathbf{u}_{t}, \mathbf{u}_{t t}\right)=\left(\mathbf{f}_{t}, \mathbf{u}_{t t}\right)
$$

Using

$$
\begin{aligned}
\left|\bar{b}\left(\mathbf{u}_{t}, \mathbf{u}, \mathbf{u}_{t t}\right)\right| & \leq C_{1}\left\|\mathbf{u}_{t}\right\|_{1}\|\mathbf{u}\|_{2}\left\|\mathbf{u}_{t t}\right\| \\
& \leq \frac{1}{8}\left\|\mathbf{u}_{t t}\right\|^{2}+C_{5}\left\|\mathbf{u}_{t}\right\|_{1}^{2}, \\
\left|\bar{b}\left(\mathbf{u}, \mathbf{u}_{t}, \mathbf{u}_{t t}\right)\right| & \leq \frac{1}{8}\left\|\mathbf{u}_{t t}\right\|^{2}+C_{5}\left\|\mathbf{u}_{t}\right\|_{1}^{2} \\
\left(\mathbf{f}_{t}, \mathbf{u}_{t t}\right) & \leq \frac{1}{4}\left\|\mathbf{u}_{t t}\right\|^{2}+\left\|\mathbf{f}_{t}\right\|^{2}, \\
\left|\left(\mathbf{u}_{t t}, A \mathbf{u}_{t}\right)\right| & \leq \frac{1}{4}\left\|\mathbf{u}_{t t}\right\|^{2}+\left\|A \mathbf{u}_{t}\right\|^{2},
\end{aligned}
$$

and combining all the above inequalities together with Lemma 4.1, we can obtain the results of this lemma.

Remark 4.1. 1. In [9], we know that for Navier-Stokes equations, if $\int_{0}^{T}\left(\left\|\mathbf{u}_{t t}\right\|^{2}+\right.$ $\left.\left\|\mathbf{u}_{t}\right\|_{2}^{2}\right) d t$ is bounded, then the following over-determined Neumann problem

$$
\begin{aligned}
& \Delta q=\operatorname{div}\left(\mathbf{f}(0)-\bar{B}\left(\mathbf{u}_{0}, \mathbf{u}_{0}\right)\right) \quad \text { in } \Omega, \\
& \left.\nabla q\right|_{\partial \Omega}=\Delta \mathbf{u}_{0}+\mathbf{f}(0)-\left.\bar{B}\left(\mathbf{u}_{0}, \mathbf{u}_{0}\right)\right|_{\partial \Omega}
\end{aligned}
$$

has solutions. For the sequential regularization formulation (2.28)-(2.30), we have a similar property. If equations (4.14)-(4.15) are solvable, we can choose the initial guess of pressure $p_{0}$ such that $p_{0}(0)$ is the solution. Let $s=1$, and rewrite equations (2.28) $-(2.30)$ by eliminating $p_{1}$ from the systems:

$$
\begin{aligned}
& \left(\mathbf{u}_{1}\right)_{t}-\frac{1}{\epsilon} \nabla \operatorname{div}\left(\left(\mathbf{u}_{1}\right)_{t}+\alpha \mathbf{u}_{1}\right)-\nu \Delta \mathbf{u}_{1}+\bar{B}\left(\mathbf{u}_{1}, \mathbf{u}_{1}\right)=\mathbf{f}-\nabla p_{0}, \\
& \left.\mathbf{u}_{1}\right|_{\partial \Omega}=0,\left.\mathbf{u}_{1}\right|_{t=0}=\mathbf{u}_{0} .
\end{aligned}
$$

Let $\mathbf{g}=\mathbf{f}(0)+\nu \Delta \mathbf{u}_{0}-\bar{B}\left(\mathbf{u}_{0}, \mathbf{u}_{0}\right)-\nabla p_{0}(0)$. Since $p_{0}(0)$ satisfies equations (4.14)(4.15), we have $\mathbf{g} \in \mathbf{H}_{0}^{1}$ and $\operatorname{div} \mathbf{g}=0$. Then following the proof of Lemma 4.3, we 
have $\operatorname{div}\left(\mathbf{u}_{1}\right)_{t}(0)=0$; hence $p_{1}(0)=p_{0}(0)$. We can then repeat this procedure to have estimations of $\mathbf{u}_{s}$ for all $s$.

2. Since the equations (4.14)-(4.15) are over-determined, we need the compatibility condition of $\mathbf{u}_{0}$ and $\mathbf{f}(0)$ to fulfil the existence of a solution. This is nonlocal and virtually uncheckable for given data. In the absence of such a compatibility condition, we will discuss another type of estimation as follows.

Lemma 4.4. Assume all the conditions as in Lemma 4.2. Moreover, $\mathbf{f}_{t} \in L^{2}\left(\mathbf{L}^{2}\right)$, and we have

$$
t\left\|\mathbf{u}_{t}\right\|_{1}^{2}+\int_{0}^{T} t\left(\left\|A \mathbf{u}_{t}\right\|^{2}+\left\|\mathbf{u}_{t t}\right\|^{2}\right) d t \leq C .
$$

As a consequence of inequality (4.16), if we fix $t_{0} \in(0, T), \forall t \geq t_{0}$,

$$
\left\|\mathbf{u}_{t}\right\|_{1}^{2}+\int_{t_{0}}^{t}\left(\frac{1}{\epsilon^{2}}\left\|\operatorname{grad} \operatorname{div} \mathbf{u}_{t t}\right\|^{2}+\frac{1}{\epsilon^{2}}\left\|\operatorname{grad} \operatorname{div} \mathbf{u}_{t}\right\|^{2}+\left\|\mathbf{u}_{t}\right\|_{2}^{2}+\left\|\mathbf{u}_{t t}\right\|^{2}\right) d t \leq C\left(t_{0}\right) .
$$

Proof. Multiplying by $A \mathbf{u}_{t}$ on both sides of equation (4.9) and estimating the trilinear form by using Young's inequality, we have

$$
\frac{1}{\epsilon}\left\|\operatorname{div} \mathbf{u}_{t t}\right\|^{2}+\frac{1}{\epsilon} \frac{d}{d t}\left\|\operatorname{div} \mathbf{u}_{t}\right\|^{2}+\frac{d}{d t}\left\|\mathbf{u}_{t}\right\|_{1}^{2}+\left\|A \mathbf{u}_{t}\right\|^{2} \leq C_{1}\left(\left\|\mathbf{u}_{t}\right\|_{1}^{2}+\left\|\mathbf{f}_{t}\right\|^{2}\right) .
$$

Multiplying by $t$ on both sides of the inequality, we obtain

$$
\frac{d}{d t} t\left(\frac{1}{\epsilon}\left\|\operatorname{div} \mathbf{u}_{t}\right\|^{2}+\left\|\mathbf{u}_{t}\right\|_{1}^{2}\right)+t\left\|A \mathbf{u}_{t}\right\|^{2} \leq\left(C_{1}+1\right)\left(\left\|\mathbf{u}_{t}\right\|_{1}^{2}+\frac{1}{\epsilon}\left\|\operatorname{div} \mathbf{u}_{t}\right\|^{2}+t\left\|\mathbf{f}_{t}\right\|^{2}\right) .
$$

Integrating both sides and recalling the inequality (4.10),

$$
\int_{0}^{T}\left(\frac{1}{\epsilon}\left\|\operatorname{div} \mathbf{u}_{t}\right\|^{2}+\left\|\mathbf{u}_{t}\right\|_{1}^{2}\right) d t \leq C,
$$

we have

$$
t\left(\frac{1}{\epsilon}\left\|\operatorname{div} \mathbf{u}_{t}\right\|^{2}+\left\|\mathbf{u}_{t}\right\|_{1}^{2}\right)+\int_{0}^{t} \tau\left\|A \mathbf{u}_{t}\right\|^{2} d \tau \leq C_{3} .
$$

Then multiplying by $\mathbf{u}_{t t}$ on both sides of equation (4.9), we have

$$
\left\|\mathbf{u}_{t t}\right\|^{2}+\left(A \mathbf{u}_{t}, \mathbf{u}_{t t}\right)+b\left(\mathbf{u}_{t}, \mathbf{u}, \mathbf{u}_{t t}\right)+b\left(\mathbf{u}, \mathbf{u}_{t}, \mathbf{u}_{t t}\right)=\left(\mathbf{f}_{t}, \mathbf{u}_{t t}\right) .
$$

Multiplying by $t$ on both sides of the equation, integrating from 0 to $t$, and noting

$$
\begin{aligned}
\left|t b\left(\mathbf{u}_{t}, \mathbf{u}, \mathbf{u}_{t t}\right)\right| & \leq C_{4}\left\|\mathbf{u}_{t}\right\|_{1}\|\mathbf{u}\|_{2}\left\|\mathbf{u}_{t t}\right\| \\
& \leq \frac{1}{8} t\left\|\mathbf{u}_{t t}\right\|^{2}+C_{5} t\left\|\mathbf{u}_{t}\right\|_{1}^{2}, \\
\left|t b\left(\mathbf{u}, \mathbf{u}_{t}, \mathbf{u}_{t t}\right)\right| & \leq \frac{1}{8} t\left\|\mathbf{u}_{t t}\right\|^{2}+C_{5} t\left\|\mathbf{u}_{t}\right\|_{1}^{2}, \\
t\left(\mathbf{f}_{t}, \mathbf{u}_{t t}\right) & \leq \frac{1}{4} t\left\|\mathbf{u}_{t t}\right\|^{2}+t\left\|\mathbf{f}_{t}\right\|^{2}, \\
\left|t\left(\mathbf{u}_{t t}, A \mathbf{u}_{t}\right)\right| & \leq \frac{1}{4} t\left\|\mathbf{u}_{t t}\right\|^{2}+t\left\|A \mathbf{u}_{t}\right\|^{2},
\end{aligned}
$$

we obtain

$$
\int_{0}^{t} \tau\left\|\mathbf{u}_{t t}\right\|^{2} d \tau \leq C
$$


Combining all the above inequalities we obtain the inequality (4.16). To obtain the inequality (4.17), we notice that $\left\|\operatorname{grad} \operatorname{div} \mathbf{u}_{t}\left(t_{0}\right)\right\| \leq C \epsilon$ (Lemma 4.2). Then applying Lemma 4.1 and replacing the initial time 0 by $t_{0}$, we obtain $\forall t>t_{0}$,

$$
\int_{t_{0}}^{t}\left(\frac{1}{\epsilon^{2}}\left\|\operatorname{grad} \operatorname{div} \mathbf{u}_{t t}\right\|^{2}+\frac{1}{\epsilon^{2}}\left\|\operatorname{grad} \operatorname{div} \mathbf{u}_{t}\right\|^{2}+\left\|\mathbf{u}_{t}\right\|_{2}^{2}\right) d t \leq C\left(\epsilon^{2}+\int_{t_{0}}^{t}\left\|A \mathbf{u}_{t}\right\|^{2} d t\right) .
$$

Then the conclusion is straightforward from inequality (4.16).

Remark 4.2. Lemma 4.3 and Lemma 4.4 are two upper bounds of the truncation error $\int_{0}^{T}\left\|\mathbf{u}_{t t}-\frac{1}{\epsilon} \operatorname{grad} \operatorname{div} \mathbf{u}_{t t}\right\|^{2} d t$. With the assumption of global compatibility, we have the energy estimation up to the initial time. Without such an assumption, the regularity remains true from a fixed small time $t_{0}$ (i.e. $t_{0}$ is a little bit away from 0 ). We will mainly use Lemma 4.3 in the next section and give a remark about the result related to Lemma 4.4

\subsection{Error estimation.}

Lemma 4.5. Let $\mathbf{f} \in L^{\infty}\left(\mathbf{L}^{\mathbf{2}}\right), \mathbf{f}_{t} \in L^{2}\left(\mathbf{L}^{2}\right), \mathbf{u}_{0} \in \mathbf{H}^{2} \cap \mathbf{H}_{0}^{1}$, div $\mathbf{u}_{0}=0$ and $\epsilon \leq \epsilon_{0}$ (as in Lemma 3.1). Then for the semi-implicit scheme (3.3), we have the estimation

$$
\left\|\mathbf{u}^{n}\right\|_{2} \leq C .
$$

Proof. Recall that we have already had a few inequalities in the previous section (see Lemma 3.3). Taking the backward difference quotient for equation (3.3), we have

$$
\begin{gathered}
\frac{1}{\triangle t}\left(1-\frac{1}{\epsilon} \operatorname{grad} \operatorname{div}\right)\left(\frac{\mathbf{u}^{n+1}-\mathbf{u}^{n}}{\triangle t}-\frac{\mathbf{u}^{n}-\mathbf{u}^{n-1}}{\triangle t}\right)-\frac{\alpha}{\epsilon} \operatorname{grad} \operatorname{div} \frac{\mathbf{u}^{n+1}-\mathbf{u}^{n}}{\triangle t} \\
-\nu \triangle \frac{\mathbf{u}^{n+1}-\mathbf{u}^{n}}{\triangle t}+\frac{\bar{B}\left(\mathbf{u}^{n}, \mathbf{u}^{n+1}\right)-\bar{B}\left(\mathbf{u}^{n-1}, \mathbf{u}^{n}\right)}{\triangle t}=\frac{\mathbf{f}^{n+1}-\mathbf{f}^{n}}{\triangle t} .
\end{gathered}
$$

Then multiplying by $\frac{\mathbf{u}^{n+1}-\mathbf{u}^{n}}{\Delta t}$ on both sides, we have

$$
\begin{aligned}
& \frac{1}{2 \triangle t}\left(\left\|\frac{\mathbf{u}^{n+1}-\mathbf{u}^{n}}{\triangle t}\right\|^{2}-\left\|\frac{\mathbf{u}^{n}-\mathbf{u}^{n-1}}{\triangle t}\right\|^{2}+\left\|\frac{\mathbf{u}^{n+1}-\mathbf{u}^{n}}{\triangle t}-\frac{\mathbf{u}^{n}-\mathbf{u}^{n-1}}{\triangle t}\right\|^{2}\right) \\
& +\frac{1}{2 \triangle t} \frac{1}{\epsilon}\left(\left\|\operatorname{div} \frac{\mathbf{u}^{n+1}-\mathbf{u}^{n}}{\triangle t}\right\|^{2}-\left\|\operatorname{div} \frac{\mathbf{u}^{n}-\mathbf{u}^{n-1}}{\triangle t}\right\|^{2}+\left\|\operatorname{div}\left(\frac{\mathbf{u}^{n+1}-\mathbf{u}^{n}}{\triangle t}-\frac{\mathbf{u}^{n}-\mathbf{u}^{n-1}}{\triangle t}\right)\right\|^{2}\right) \\
& +\frac{\alpha}{\epsilon}\left\|\operatorname{div} \frac{\mathbf{u}^{n+1}-\mathbf{u}^{n}}{\triangle t}\right\|^{2}+\nu\left\|\operatorname{grad} \frac{\mathbf{u}^{n+1}-\mathbf{u}^{n}}{\triangle t}\right\|^{2}+\frac{1}{\triangle t}\left(\bar{b}\left(\mathbf{u}^{n}, \mathbf{u}^{n+1}, \frac{\mathbf{u}^{n+1}-\mathbf{u}^{n}}{\triangle t}\right)\right. \\
& \left.\quad-\bar{b}\left(\mathbf{u}^{n-1}, \mathbf{u}^{n}, \frac{\mathbf{u}^{n+1}-\mathbf{u}^{n}}{\triangle t}\right)\right)=\left(\frac{\mathbf{f}^{n+1}-\mathbf{f}^{n}}{\triangle t}, \frac{\mathbf{u}^{n+1}-\mathbf{u}^{n}}{\triangle t}\right) .
\end{aligned}
$$

Since $\bar{b}$ is a trilinear form, we have

$$
\begin{aligned}
& \frac{1}{\triangle t}\left(\bar{b}\left(\mathbf{u}^{n}, \mathbf{u}^{n+1}, \frac{\mathbf{u}^{n+1}-\mathbf{u}^{n}}{\triangle t}\right)-\bar{b}\left(\mathbf{u}^{n-1}, \mathbf{u}^{n}, \frac{\mathbf{u}^{n+1}-\mathbf{u}^{n}}{\triangle t}\right)\right) \\
& \quad=\bar{b}\left(\mathbf{u}^{n}, \frac{\mathbf{u}^{n+1}-\mathbf{u}^{n}}{\triangle t}, \frac{\mathbf{u}^{n+1}-\mathbf{u}^{n}}{\triangle t}\right)-\bar{b}\left(\frac{\mathbf{u}^{n}-\mathbf{u}^{n-1}}{\triangle t}, \mathbf{u}^{n}, \frac{\mathbf{u}^{n+1}-\mathbf{u}^{n}}{\triangle t}\right) .
\end{aligned}
$$


Since

$$
\begin{gathered}
\bar{b}\left(\mathbf{u}^{n}, \frac{\mathbf{u}^{n+1}-\mathbf{u}^{n}}{\triangle t}, \frac{\mathbf{u}^{n+1}-\mathbf{u}^{n}}{\triangle t}\right)=0, \\
\left|\bar{b}\left(\frac{\mathbf{u}^{n}-\mathbf{u}^{n-1}}{\triangle t}, \mathbf{u}^{n}, \frac{\mathbf{u}^{n+1}-\mathbf{u}^{n}}{\triangle t}\right)\right| \\
\leq C_{1}\left\|\mathbf{u}^{n}\right\|_{2}\left\|\frac{\mathbf{u}^{n+1}-\mathbf{u}^{n}}{\triangle t}\right\|_{1}\left\|\frac{\mathbf{u}^{n}-\mathbf{u}^{n-1}}{\triangle t}\right\| \\
\leq \delta\left\|\frac{\mathbf{u}^{n+1}-\mathbf{u}^{n}}{\triangle t}\right\|_{1}^{2}+\frac{C_{1}}{\delta}\left\|\mathbf{u}^{n}\right\|_{2}^{2}\left\|\frac{\mathbf{u}^{n}-\mathbf{u}^{n-1}}{\triangle t}\right\|^{2},
\end{gathered}
$$

and

$$
\left(\frac{\mathbf{f}^{n+1}-\mathbf{f}^{n}}{\triangle t}, \frac{\mathbf{u}^{n+1}-\mathbf{u}^{n}}{\triangle t}\right) \leq \delta\left\|\frac{\mathbf{u}^{n+1}-\mathbf{u}^{n}}{\triangle t}\right\|_{1}^{2}+\frac{1}{\delta}\left\|\frac{\mathbf{f}^{n+1}-\mathbf{f}^{n}}{\triangle t}\right\|^{2},
$$

we can choose $\delta$ to be properly small, i.e. $\delta\left\|\frac{\mathbf{u}^{n+1}-\mathbf{u}^{n}}{\Delta t}\right\|_{1}^{2} \leq \frac{\nu}{4}\left\|\operatorname{grad} \frac{\mathbf{u}^{n+1}-\mathbf{u}^{n}}{\Delta t}\right\|^{2}$. Defining $y^{n+1}=\left\|\frac{\mathbf{u}^{n+1}-\mathbf{u}^{n}}{\Delta t}\right\|^{2}+\frac{1}{\epsilon}\left\|\operatorname{div} \frac{\mathbf{u}^{n+1}-\mathbf{u}^{n}}{\Delta t}\right\|^{2}$, we have

$$
\begin{gathered}
\frac{1}{\triangle t}\left(y^{n+1}-y^{n}\right)+\frac{1}{\epsilon}\left\|\operatorname{div} \frac{\mathbf{u}^{n+1}-\mathbf{u}^{n}}{\triangle t}\right\|^{2}+\left\|\frac{\mathbf{u}^{n+1}-\mathbf{u}^{n}}{\triangle t}\right\|_{1}^{2} \\
\leq C_{2}\left(\left\|\mathbf{u}^{n}\right\|_{2}^{2}\left(y^{n+1}+y^{n}\right)+\left\|\frac{\mathbf{f}^{n+1}-\mathbf{f}^{n}}{\triangle t}\right\|^{2}\right) .
\end{gathered}
$$

Hence, $\left\|\frac{\mathbf{f}^{n+1}-\mathbf{f}^{n}}{\Delta t}\right\|^{2} \leq \frac{1}{\Delta t} \int_{t_{n-1}}^{t_{n+1}}\left\|\mathbf{f}_{t}\right\|^{2} d t$ due to $\frac{\mathbf{f}^{n+1}-\mathbf{f}^{n}}{\Delta t}=\frac{1}{\Delta t} \int_{t_{n}}^{t_{n+1}} \mathbf{f}_{t} d t$. Summing for $n$, we get

$$
\triangle t \sum_{n=0}^{N-1}\left\|\frac{\mathbf{f}^{n+1}-\mathbf{f}^{n}}{\triangle t}\right\|^{2} \leq \int_{0}^{T}\left\|\mathbf{f}_{t}\right\|^{2} d t .
$$

Since we already had that $\triangle t \sum_{n=0}^{N}\left\|\mathbf{u}^{n}\right\|_{2}^{2}$ is bounded, summing (4.20) for $n$ and applying the discrete Gronwall's inequality, we obtain $y^{n} \leq C_{3}\left(\int_{0}^{T}\left\|\mathbf{f}_{t}\right\|^{2} d t+y^{1}\right)$. We still need to estimate $y^{1}=\left\|\frac{\mathbf{u}^{1}-\mathbf{u}^{0}}{\Delta t}\right\|^{2}+\frac{1}{\epsilon}\left\|\operatorname{div} \frac{\mathbf{u}^{1}-\mathbf{u}^{0}}{\Delta t}\right\|^{2}$. Multiplying by $\frac{\mathbf{u}^{n+1}-\mathbf{u}^{n}}{\Delta t}$ on both sides of equation (3.3) and letting $n=0$, we have

$$
y^{1}+\frac{\alpha}{\epsilon}\left(\operatorname{div} \mathbf{u}^{1}, \operatorname{div} \frac{\mathbf{u}^{1}-\mathbf{u}^{0}}{\triangle t}\right)+\nu\left(\operatorname{grad} \mathbf{u}^{1}, \operatorname{grad} \frac{\mathbf{u}^{1}-\mathbf{u}^{0}}{\triangle t}\right)+\bar{b}\left(\mathbf{u}^{0}, \mathbf{u}^{1}, \frac{\mathbf{u}^{1}-\mathbf{u}^{0}}{\triangle t}\right)=\left(\mathbf{f}^{1}, \frac{\mathbf{u}^{1}-\mathbf{u}^{0}}{\triangle t}\right) .
$$

Noting $\operatorname{div} \mathbf{u}^{0}=0\left(\right.$ since $\left.\mathbf{u}^{0}=\mathbf{u}_{0}\right)$ and $\left(\operatorname{grad} \mathbf{u}^{1}, \operatorname{grad} \frac{\mathbf{u}^{1}-\mathbf{u}^{0}}{\Delta t}\right)=\frac{1}{\Delta t} \| \operatorname{grad}\left(\mathbf{u}^{1}-\right.$ $\left.\mathbf{u}^{0}\right) \|^{2}-\left(\triangle \mathbf{u}^{0}, \frac{\mathbf{u}^{1}-\mathbf{u}^{0}}{\triangle t}\right)$,

$$
\begin{aligned}
\left|\left(\triangle \mathbf{u}^{0}, \frac{\mathbf{u}^{1}-\mathbf{u}^{0}}{\triangle t}\right)\right| & \leq \frac{1}{4} y^{1}+C_{4}\left\|\mathbf{u}^{0}\right\|_{2}^{2}, \\
\left|\left(\mathbf{f}^{1}, \frac{\mathbf{u}^{1}-\mathbf{u}^{0}}{\triangle t}\right)\right| & \leq \frac{1}{4} y^{1}+\left\|\mathbf{f}^{1}\right\|^{2}, \\
\left|\bar{b}\left(\mathbf{u}^{0}, \mathbf{u}^{1}, \frac{\mathbf{u}^{1}-\mathbf{u}^{0}}{\triangle t}\right)\right| & \leq C_{1}\left\|\mathbf{u}^{0}\right\|_{2}\left\|\mathbf{u}^{1}\right\|_{1}\left\|\frac{\mathbf{u}^{1}-\mathbf{u}^{0}}{\triangle t}\right\| \\
& \leq \frac{1}{4} y^{1}+C_{1}\left\|\mathbf{u}^{0}\right\|_{2}^{2}\left\|\mathbf{u}^{1}\right\|_{1}^{2},
\end{aligned}
$$

and that $\left\|\mathbf{u}^{0}\right\|_{2}$ and $\left\|\mathbf{u}^{1}\right\|_{1}$ are bounded, we obtain $y^{1} \leq C_{5}$. Hence, $y^{n} \leq C_{6}$. Multiplying by $A \mathbf{u}^{n+1}$ on both sides of equation (3.3), where the operator $A$ is defined in Lemma 3.1, we have

$$
\left(\frac{\mathbf{u}^{n+1}-\mathbf{u}^{n}}{\triangle t}, A \mathbf{u}^{n+1}\right)+\left\|A \mathbf{u}^{n+1}\right\|^{2}+\bar{b}\left(\mathbf{u}^{n}, \mathbf{u}^{n+1}, A \mathbf{u}^{n+1}\right)=\left(\mathbf{f}^{n+1}, A \mathbf{u}^{n+1}\right) .
$$


Since

$$
\begin{gathered}
\left|\left(\frac{\mathbf{u}^{n+1}-\mathbf{u}^{n}}{\Delta t}, A \mathbf{u}^{n+1}\right)\right| \leq \frac{1}{4}\left\|A \mathbf{u}^{n+1}\right\|^{2}+y^{n}, \\
\left|\left(\mathbf{f}^{n+1}, A \mathbf{u}^{n+1}\right)\right| \leq \frac{1}{4}\left\|A \mathbf{u}^{n+1}\right\|^{2}+\left\|\mathbf{f}^{n+1}\right\|^{2}
\end{gathered}
$$

and

$$
\begin{aligned}
\left|\bar{b}\left(\mathbf{u}^{n}, \mathbf{u}^{n+1}, A \mathbf{u}^{n+1}\right)\right| & \leq C_{7}\left\|\mathbf{u}^{n}\right\|_{1}\left\|\mathbf{u}^{n+1}\right\|_{1}^{\frac{1}{2}}\left\|\mathbf{u}^{n+1}\right\|_{2}^{\frac{1}{2}}\left\|A \mathbf{u}^{n+1}\right\| \\
& \leq \frac{1}{4}\left\|A \mathbf{u}^{n+1}\right\|^{2}+\delta\left\|\mathbf{u}^{n+1}\right\|_{2}^{2}+\frac{C_{8}}{\delta},
\end{aligned}
$$

choosing a properly small $\delta$ and using Lemma 3.1 and the boundedness of $y^{n}$, we can conclude that $A \mathbf{u}^{n}$ is uniformly bounded. Therefore, $\left\|\mathbf{u}^{n}\right\|_{2} \leq C$ from Lemma 3.1 .

Theorem 4.6. For the semi-implicit scheme, defining the error function $\mathbf{e}^{n}=$ $\mathbf{u}^{n}-\mathbf{u}\left(t_{n}\right)$, then with the same assumption as in Lemma 4.3, we have

$$
\left\|\mathbf{e}^{n}\right\|_{1}^{2}+\triangle t \sum_{0}^{N}\left(\frac{1}{\epsilon^{2}}\left\|\operatorname{grad} \operatorname{div} \mathbf{e}^{n}\right\|+\left\|\mathbf{e}^{n}\right\|_{2}^{2}\right) \leq C \triangle t^{2} .
$$

Proof. The error function $\mathbf{e}^{n}$ satisfies the equation:

$$
\begin{gathered}
\frac{\mathbf{e}^{n+1}-\mathbf{e}^{n}}{\triangle t}-\frac{1}{\epsilon} \operatorname{grad} \operatorname{div}\left(\frac{\mathbf{e}^{n+1}-\mathbf{e}^{n}}{\triangle t}+\alpha \mathbf{e}^{n+1}\right)-\nu \triangle \mathbf{e}^{n+1} \\
+\bar{B}\left(\mathbf{u}^{n}, \mathbf{u}^{n+1}\right)-\bar{B}\left(\mathbf{u}\left(t_{n+1}\right), \mathbf{u}\left(t_{n+1}\right)\right)=\mathbf{r}^{n+1} .
\end{gathered}
$$

The difference of the nonlinear terms can be rewritten as:

$\bar{B}\left(\mathbf{u}^{n}, \mathbf{u}^{n+1}\right)-\bar{B}\left(\mathbf{u}\left(t_{n+1}\right), \mathbf{u}\left(t_{n+1}\right)\right)=\bar{B}\left(\mathbf{u}^{n}-\mathbf{u}^{n+1}, \mathbf{u}^{n+1}\right)+\bar{B}\left(\mathbf{u}^{n+1}, \mathbf{e}^{n+1}\right)+\bar{B}\left(\mathbf{e}^{n+1}, \mathbf{u}\left(t_{n+1}\right)\right)$.

Multiplying by $\mathbf{e}^{n+1}$ on both sides of equation (4.22), we now estimate the nonlinear part term by term:

$$
\begin{gathered}
\left|\bar{b}\left(\mathbf{u}^{n}-\mathbf{u}^{n+1}, \mathbf{u}^{n+1}, \mathbf{e}^{n+1}\right)\right| \begin{array}{l}
\leq C_{1}\left\|\mathbf{u}^{n}-\mathbf{u}^{n+1}\right\|\left\|\mathbf{u}^{n+1}\right\|_{2}\left\|\mathbf{e}^{n+1}\right\|_{1} \\
\leq \delta\left\|\mathbf{e}^{n+1}\right\|_{1}^{2}+\frac{C_{2}}{\delta}\left\|\mathbf{u}^{n}-\mathbf{u}^{n+1}\right\|^{2}
\end{array} \\
\bar{b}\left(\mathbf{u}^{n+1}, \mathbf{e}^{n+1}, \mathbf{e}^{n+1}\right)=0 \\
\left|\bar{b}\left(\mathbf{e}^{n+1}, \mathbf{u}\left(t_{n+1}\right), \mathbf{e}^{n+1}\right)\right| \leq \delta\left\|\mathbf{e}^{n+1}\right\|_{1}^{2}+\frac{C_{2}}{\delta}\left\|\mathbf{e}^{n+1}\right\|^{2} .
\end{gathered}
$$

Choosing a properly small $\delta$, i.e. $\delta\left\|\mathbf{e}^{n+1}\right\|_{1}^{2} \leq \frac{1}{4}\left\|\operatorname{grade}^{n+1}\right\|^{2}$, and defining $y^{n}=$ $\left\|\mathbf{e}^{n}\right\|^{2}+\frac{1}{\epsilon}\left\|\operatorname{div}^{n}\right\|^{2}$, we have

$\frac{1}{\triangle t}\left(y^{n+1}-y^{n}\right)+\frac{1}{\epsilon}\left\|\operatorname{dive}^{n+1}\right\|^{2}+\left\|\mathbf{e}^{n+1}\right\|_{1}^{2} \leq C_{3}\left(\left\|\mathbf{r}^{n+1}\right\|^{2}+\left\|\mathbf{u}^{n+1}-\mathbf{u}^{n}\right\|^{2}+y^{n+1}\right)$.

Noting that $\triangle t \sum\left\|\mathbf{r}^{n}\right\|^{2} \leq C \triangle t^{2}$ (see inequality (4.2) and Lemma 4.4) and $\triangle t \sum\left\|\mathbf{u}^{n+1}-\mathbf{u}^{n}\right\|^{2} \leq C \triangle t^{2}$ (see Lemma 3.3) and applying the discrete Gronwall's inequality we obtain

$$
y^{n}+\triangle t \sum_{0}^{n}\left(\frac{1}{\epsilon}\left\|\operatorname{dive} \mathbf{e}^{i}\right\|^{2}+\left\|\mathbf{e}^{i}\right\|_{1}^{2}\right) \leq C_{4}\left(y^{0}+\triangle t^{2}\right) .
$$

Since $\mathbf{u}^{0}=\mathbf{u}(0)$, we then have $y^{0}=0$; hence

$$
\left\|\mathbf{e}^{n}\right\|^{2}+\triangle t \sum_{0}^{N}\left\|\mathbf{e}^{n}\right\|_{1}^{2} \leq C \triangle t^{2} .
$$


To obtain a stronger error estimation, we need to multiply by $A \mathbf{e}^{n+1}$ on both sides of equation (4.22) and have

$$
\begin{aligned}
& \left(\frac{\mathbf{e}^{n+1}-\mathbf{e}^{n}}{\triangle t}, A \mathbf{e}^{n+1}\right)+\left\|A \mathbf{e}^{n+1}\right\|^{2}+\bar{b}\left(\mathbf{u}^{n}-\mathbf{u}^{n+1}, \mathbf{u}^{n+1}, A \mathbf{e}^{n+1}\right) \\
& \quad+\bar{b}\left(\mathbf{u}^{n+1}, \mathbf{e}^{n+1}, A \mathbf{e}^{n+1}\right)+\bar{b}\left(\mathbf{e}^{n+1}, \mathbf{u}\left(t_{n+1}\right), A \mathbf{e}^{n+1}\right)=\left(\mathbf{r}^{n+1}, A \mathbf{e}^{n+1}\right) .
\end{aligned}
$$

We now deal with the above equation term by term:

$$
\begin{gathered}
\left(\frac{\mathbf{e}^{n+1}-\mathbf{e}^{n}}{\triangle t}, A \mathbf{e}^{n+1}\right)=\frac{1}{\epsilon}\left\|\operatorname{div} \frac{\mathbf{e}^{n+1}-\mathbf{e}^{n}}{\triangle t}\right\|^{2}+\frac{\alpha}{2 \epsilon \triangle t}\left(\left\|\operatorname{div} \mathbf{e}^{n+1}\right\|^{2}-\left\|\operatorname{div} \mathbf{e}^{n}\right\|^{2}\right. \\
\left.+\left\|\operatorname{div}\left(\mathbf{e}^{n+1}-\mathbf{e}^{n}\right)\right\|^{2}\right)+\frac{\nu}{2 \triangle t}\left(\left\|\operatorname{grade}^{n+1}\right\|^{2}-\left\|\operatorname{grad} \mathbf{e}^{n}\right\|^{2}+\left\|\operatorname{grad}\left(\mathbf{e}^{n+1}-\mathbf{e}^{n}\right)\right\|^{2}\right), \\
\left|\bar{b}\left(\mathbf{u}^{n}-\mathbf{u}^{n+1}, \mathbf{u}^{n+1}, A \mathbf{e}^{n+1}\right)\right| \leq \frac{1}{8}\left\|A \mathbf{e}^{n+1}\right\|^{2}+C_{5}\left\|\mathbf{u}^{n}-\mathbf{u}^{n+1}\right\|_{1}^{2}\left\|\mathbf{u}^{n+1}\right\|_{1}^{2}, \\
\left|\bar{b}\left(\mathbf{u}^{n+1}, \mathbf{e}^{n+1}, A \mathbf{e}^{n+1}\right)\right| \leq \frac{1}{8}\left\|A \mathbf{e}^{n+1}\right\|^{2}+C_{5}\left\|\mathbf{e}^{n+1}\right\|_{1}^{2}, \\
\left|\bar{b}\left(\mathbf{e}^{n+1}, \mathbf{u}\left(t_{n+1}\right), A \mathbf{e}^{n+1}\right)\right| \leq \frac{1}{8}\left\|A \mathbf{e}^{n+1}\right\|^{2}+C_{5}\left\|\mathbf{e}^{n+1}\right\|_{1}^{2}, \\
\left|\left(\mathbf{r}^{n+1}, A \mathbf{e}^{n+1}\right)\right| \leq \frac{1}{8}\left\|A \mathbf{e}^{n+1}\right\|^{2}+2\left\|\mathbf{r}^{n+1}\right\|^{2} .
\end{gathered}
$$

Defining $y^{n}=\frac{1}{\epsilon}\left\|\operatorname{div} \mathbf{e}^{n}\right\|+\left\|\mathbf{e}^{n}\right\|_{1}^{2}$ and using the above inequalities, we have

$$
\frac{\left(y^{n+1}-y^{n}\right)}{\triangle t}+\left\|A \mathbf{e}^{n+1}\right\|^{2} \leq C_{6}\left(\left\|\mathbf{u}^{n}-\mathbf{u}^{n+1}\right\|_{1}^{2}+\left\|\mathbf{e}^{n+1}\right\|_{1}^{2}+\left\|\mathbf{r}^{n+1}\right\|^{2}\right) .
$$

From the inequality (4.20), we obtain $\triangle t \sum\left\|\mathbf{u}^{n}-\mathbf{u}^{n+1}\right\|_{1}^{2} \leq C \triangle t^{2}$. We have just derived $\triangle t \sum\left\|\mathbf{e}^{n}\right\|_{1}^{2} \leq C \triangle t^{2}$ in (4.23). From condition $\triangle t \sum\left\|\mathbf{r}^{n}\right\|^{2} \leq C \triangle t^{2}$, applying the discrete Gronwall's inequality, using $\mathbf{e}^{0}=0$ and Lemma 3.1 we have

$$
\left\|\mathbf{e}^{n}\right\|_{1}^{2}+\triangle t \sum_{0}^{N}\left(\frac{1}{\epsilon^{2}}\left\|\operatorname{grad} \operatorname{div} \mathbf{e}^{n}\right\|^{2}+\left\|\mathbf{e}^{n}\right\|_{2}^{2}\right) \leq C \triangle t^{2} .
$$

We apply the semi-implicit scheme to the equations (2.28)-(2.30). Let the solution of the time discrete scheme be $\mathbf{u}_{s}^{n}$ and the solution of the Navier-Stokes equations be $(\mathbf{u}, p)$. If the initial value $\mathbf{u}_{0}$ and $\mathbf{f}(0)$ satisfy the global compatibility condition such that the overdetermined Neumann problem (4.14)-(4.15) has a solution, then we can choose our initial guess $p_{0}(0)$ to solve this equation with $\int_{\Omega} p_{0}(0) d \mathbf{x}=0$. Combining Theorem 2.5 and Theorem 4.6. we have our main theorem.

Theorem 4.7. Let $\mathbf{f} \in L^{\infty}\left(\mathbf{L}^{2}\right), \mathbf{f}_{t} \in L^{2}\left(\mathbf{L}^{2}\right), \mathbf{u}_{0} \in \mathbf{H}^{2} \cap \mathbf{H}_{0}^{1}$, div $\mathbf{u}_{0}=0$, $\epsilon$ be less than a constant $\epsilon_{0}$, the initial guess satisfy $\int_{0}^{T}\left\|p_{0}\right\|_{1}^{2} d t \leq 2+2 \int_{0}^{T}\|p\|_{1}^{2} d t$ and $p_{0}(0)$ solve equation (4.14) 4.15. Then we have

$$
\left\|\mathbf{u}_{s}^{n}-\mathbf{u}\left(t_{n}\right)\right\|_{1}^{2}+\triangle t \sum_{0}^{n}\left(\frac{1}{\epsilon^{2}}\left\|\operatorname{graddiv}\left(\mathbf{u}_{s}^{i}-\mathbf{u}\left(t_{i}\right)\right)\right\|^{2}+\left\|\mathbf{u}_{s}^{i}-\mathbf{u}\left(t_{i}\right)\right\|_{2}^{2}\right) \leq C\left(\triangle t^{2}+\left(M \epsilon^{2}\right)^{s}\right) .
$$

Remark 4.3. We use $\triangle t \sum\left\|\mathbf{r}^{n}\right\|^{2} \leq C \triangle t^{2}$ in the proof. Without a global compatibility assumption, this may not hold in general. But we should have $\triangle t \sum_{n_{0}}^{N}\left\|\mathbf{r}^{n}\right\|^{2} \leq$ $C\left(n_{0} \triangle t\right) \triangle t^{2}$, where the constant $C$ may depend on $n_{0} \triangle t$. In this case, after a small fixed time, the error estimation remains true. 
Remark 4.4. In practice we are also interested in the fully explicit treatment of the nonlinear convection term, i.e. the scheme (3.4). If we assume a priori estimation of $\mathbf{u}^{n}$, i.e. $\left\|\mathbf{u}^{n}\right\|$ is uniformly bounded, we can have a similar result as in Theorem 4.7. This assumption of uniform boundedness of $\left\|\mathbf{u}^{n}\right\|$ is technically difficult to be generally verified for the scheme (3.4) up to a given time (see Lemma 3.5 for the boundedness of the short time solution $\left.\left\|\mathbf{u}^{n}\right\|\right)$. But it is often true in physical or practical examples. We can also consider other schemes in time, for example, the fully implicit scheme or the Crank-Nicolson scheme as in 14. The results will be similar; i.e., with some global compatibility assumption, we have error estimations of Theorem 4.7 up to the initial time; without it the results remain true after a small fixed time.

\section{REFERENCES}

[1] C. Amrouche and V. Girault, Decomposition of vector spaces and application to the Stokes problem in arbitrary dimension, Czechoslovak Mathematical J. 44 (1994), No. 119, pp. 109140. MR.1257940 (95c:35190)

[2] U. Ascher and P. Lin, Sequential regularization methods for higher index DAEs with constraint singularities: Linear index-2 case, SIAM J. Numer. Anal. 33 (1996). 1921-1940. MR.1411855 (98c:65133)

[3] U. M. Ascher and P. Lin, Sequential regularization methods for nonlinear higher index DAEs, SIAM J. Sci. Comput. 18 (1997), no.1, 160-181. MR.1433382 (97k:65180)

[4] J. Baumgarte, Stabilization of constraints and integrals of motion in dynamical systems, Comp. Meth. in Appl. Mech. and Eng. 1 (1972), 1-16. MR0391628 (52:12448)

[5] L. C. Evans, Partial Differential Equations. American Mathematical Society, 1998. MR.1625845 (99e:35001)

[6] M. Fortin and R. Glowinski, Augmented Lagrangian Methods: Applications to the Numerical Solution of Boundary-Value Problems, Studies in Mathematics and Its Applications 15, North-Holland, 1983. MR724072 (85a:49004)

[7] O. Gonzalez, J.H. Maddocks and R.L. Pego, Multi-multiplier ambient-space formulations of constrained dynamical systems, with an application to elastodynamics, Arch. Rational Mech. Anal. 157 (2001) 285-323. MR.1831174 (2002k:74027)

[8] K. Gustafson, Theory and computation of periodic solutions of autonomous partial differential equation boundary value problems, with application to the driven cavity problem, Math. Comput. Modelling 22 (1995), No. 9, 57-75. MR.1365111 (96h:76023)

[9] J.G. Heywood and R. Rannacher, Finite element approximation of the nonstationary NavierStokes problem, I. Regularity of solutions and second-order error estimates for spatial discretization, SIAM J. Numer. Anal. 19 (1982), 275-311. MR650052 (83d:65260)

[10] P. Lin, A sequential regularization method for time-dependent incompressible Navier-Stokes equations, SIAM J. Numer. Anal. 34 (1997), no.3, 1051-1071. MR1451113 (98b:65110)

[11] P. Lin, Regularization Methods for Differential Equations and Their Numerical Solution, Ph.D. thesis, Department of Mathematics, University of British Columbia, 1995.

[12] P. Lin, Q. Guo and X. Chen, A fully explicit method and its parallelization for incompressible flow computation, Comput. Methods Appl. Mech. Engrg. 192/22-24 (2003), 2555-2564.

[13] J. G. Liu, J. Liu, R. L. Pego, Stability and convergence of efficient Navier-Stokes solvers via a commutator estimate, Comm. Pure Appl. Math. 60 (2007), pp. 1443-1487. MR2342954

[14] Lu Xiliang, Error Analysis for Incompressible Viscous Flow Based on a Sequential Regularization Formulation, Ph.D. thesis, Department of Mathematics, National University of Singapore, 2006.

[15] R. Rannacher, On the numerical solution of the incompressible Navier-Stokes equations, Z. Angew. Math. Mech. 73 (1993), pp. 203-216. MR1242221 (94h:76073)

[16] J. Shen, Hopf bifurcation of the unsteady regularized driven cavity flows, J. Comp. Phy. 95 (1991), 228-245.

[17] J. Shen, On error estimates of the penalty method for the unsteady Navier-Stokes equations, SIAM J. Numer. Anal. 32 (1995), pp. 386-403. MR1324294 (96d:65153) 
[18] R. Temam, Navier-Stokes Equations, North-Holland, Amsterdam, 1977. MR769654 (86m:76003)

[19] R. Temam, Navier-Stokes Equations and Nonlinear Functional Analysis, 2nd edition, CBMSNSF Regional Conference Series in Applied Mathematics, 1995. MR1318914 (96e:35136)

Department of Mathematics, National University of Singapore, Science Drive 2, Singapore 117543 , Singapore

Current address: Johann Radon Institute for Computational and Applied Mathematics (RICAM), Austrian Academy of Sciences, Altenberger Strasse 69, A-4040 Linz, Austria

E-mail address: lu_xiliang@hotmail.com

Department of Mathematics, National University of Singapore, Science Drive 2, Singapore 117543 , Singapore

E-mail address: matlinp@nus.edu.sg

Department of Mathematics, University of Maryland, College Park, Maryland $20742-4015$

E-mail address: jliu@math.umd.edu 